\title{
Rapidly varying boundaries in equations with nonlinear boundary conditions. The case of a Lispchitz deformation.
}

\author{
José M. ARRIETA*and Simone M. BRUSCHI ${ }^{\dagger}$ \\ * Dept. Matemática Aplicada, Univ. Complutense, 28040 Madrid, Spain, [jose.arrieta@mat.ucm.es] \\ $\dagger$ Dept. Matemática, Univ. Estadual Paulista Rio Claro - SP, Brazil, [sbruschi@rc.unesp.br]
}

January 25, 2007

\section{Introduction}

This article is devoted to the study of the behavior of the solutions of an elliptic equation with nonlinear boundary conditions of the type

$$
\begin{cases}-\Delta u+u=f(x, u), & \text { in } \Omega_{\epsilon} \\ \frac{\partial u}{\partial n}+g(x, u)=0, & \text { on } \partial \Omega_{\epsilon} .\end{cases}
$$

when the boundary of the domain varies very rapidly as a parameter $\epsilon \rightarrow 0$. We consider a family of uniformly bounded smooth domains $\Omega_{\epsilon} \subset \mathbb{R}^{N}, 0 \leq \epsilon \leq \epsilon_{0}$, which satisfy both $\Omega_{\epsilon} \rightarrow \Omega \equiv \Omega_{0}$ and $\partial \Omega_{\epsilon} \rightarrow \partial \Omega$ in the sense of Hausdorff, that is $\operatorname{dist}\left(\Omega_{\epsilon}, \Omega\right)+\operatorname{dist}\left(\partial \Omega_{\epsilon}, \partial \Omega\right) \rightarrow 0$ as $\epsilon \rightarrow 0$, where dist is the symmetric Hausdorff distance of two sets in $\mathbb{R}^{N}(\operatorname{dist}(A, B)=$ $\left.\sup _{x \in A} \inf _{y \in B}|x-y|+\sup _{y \in B} \inf _{x \in A}|x-y|\right)$. Observe that this setting does not allow the possibility of perforated domains. We will look at this problem from the perturbation of domain point of view and we refer to $\Omega$ as the unperturbed domain and $\Omega_{\epsilon}$ as the perturbed domains. We also assume that the nonlinearities $f, g: U \times \mathbb{R} \rightarrow \mathbb{R}$ are continuous in both variables and $C^{2}$ in the second one where $U$ is a fixed and smooth bounded domain containing all $\bar{\Omega}_{\epsilon}$, for all $0 \leq \epsilon \leq \epsilon_{0}$.

Although the domains behave continuously as $\epsilon \rightarrow 0$, the way in which the boundary $\partial \Omega_{\epsilon}$ approach $\partial \Omega$ may not be smooth. In particular, this setting includes the case where the

\footnotetext{
*Partially supported by Project BFM2003-03810 DGES, Spain.

${ }^{\dagger}$ Partially supported by FAPESP proc. 04/06020-4 Brazil and BFM2003-03810 DGES, Spain.
} 
boundary $\partial \Omega_{\epsilon}$ presents a highly oscillatory behavior as $\epsilon \rightarrow 0$, as it is the case of boundary homogenization problems. The interaction of a highly oscillatory behavior of the boundary and the nonlinear boundary condition in equation (1.1) is the main objective of the present work. The results of this paper and of the continuation of this one, see [3], where announced in 2 .

In the present paper we will consider a class of boundary perturbation which, roughly speaking, is characterized by the fact that locally around each point $x_{0} \in \partial \Omega$ and for all $0<r \leq 1$, we have $\frac{\left|\partial \Omega_{\epsilon} \cap B\left(x_{0}, r\right)\right|}{\left|\partial \Omega \cap B\left(x_{0}, r\right)\right|} \leq C$, for some constant $C$ independent of $x_{0}, r$ and $\epsilon$, where we denote by $|\cdot|$ the $(N-1)$-dimensional measure. Observe that this is the case for instance if $N=2$ and $\partial \Omega$ is written locally around certain point as the graph of a function $y=\varphi(x)$ and $\partial \Omega_{\epsilon}$ is written locally around the same point as the graph of the function $y=\varphi(x)+\epsilon \sin \left(x \epsilon^{-\alpha}\right)$ for some $0 \leq \alpha \leq 1$. Actually, the fact that $\alpha \leq 1$ guarantees that the quotient above is bounded by a constant $C$. Moreover, if $0<\alpha<1$ then $C$ can be chosen arbitrarily close to 1 for $\epsilon$ small enough but if $\alpha=1$, then $C$ is not close to 1 even for $\epsilon$ small. In the subsequent paper, [3], we will treat the case where we allow $\frac{\left|\partial \Omega_{\epsilon} \cap B\left(x_{0}, r\right)\right|}{\left|\partial \Omega \cap B\left(x_{0}, r\right)\right|} \rightarrow+\infty$, which is the case of $\alpha>1$ in the example above.

As a matter of fact, the behavior of the quotient above as $\epsilon \rightarrow 0$ is of fundamental importance when trying to understand the limit behavior of problem (1.1) when $\epsilon \rightarrow 0$. We will see that under certain conditions, the limiting equation of (1.1) is given by

$$
\begin{cases}-\Delta u+u=f(x, u), & \text { in } \Omega \\ \frac{\partial u}{\partial n}+\gamma(x) g(x, u)=0, & \text { on } \partial \Omega .\end{cases}
$$

where the function $\gamma$ is related to the behavior of the quotient above and it satisfies $\gamma \in$ $L^{\infty}(\partial \Omega)$ and $\gamma \geq 1$. The precise hypotheses on the domains and the exact definition of the function $\gamma$ are stated in hypotheses $(\mathbf{H})$ and $(\mathbf{F})$ in Section 2.

We will be able to prove a "convergence result". Actually, we will show that the solutions of (1.1) converge to the solutions of (1.2) in $H^{1}$ and also in $C^{\beta}$, for some $\beta>0$. Moreover, we will prove that the convergence of equilibria is also obtained in terms of the spectra of the linearizations of the equations around the equilibria, that is, if $u_{\epsilon}^{*}$ is a sequence of equilibria of (1.1) which converge to $u_{0}^{*}$, an equilibrium of (1.2), then the eigenvalues and eigenfunctions of the linearization of (1.1) around $u_{\epsilon}^{*}$ converge to the eigenvalues and eigenfunctions of the linearization of (1.2) around $u_{0}^{*}$. Also, we will prove a "uniqueness result", in the sense that for any hyperbolic equilibrium of the limiting problem (1.2), (hyperbolic in the sense that the eigenvalues of the linearized operator of (1.2) around the equilibrium do not intersect the imaginary axis), then there exists one and only one equilibrium of (1.1) in its neighborhood. In particular, if all the equilibria of (1.2) are hyperbolic, then there exists only a finite number of them and for all $\epsilon$ small enough, problem (1.1) has exactly the same number of equilibria and they are close to the equilibria of (1.2). These results are stated in Theorem 2.4 and Theorem 2.6] in Section 2.

The behavior of solutions of elliptic partial differential equations in the presence of bound- 
ary oscillations is a subject that has been addressed in the literature by different authors. We would like to mention [18 for a general reference of homogenization, including boundary homogenization. Also, the work [1] deals with boundary homogenization with different boundary conditions and the coefficients appearing in the boundary condition depend also on the parameter $\epsilon$. In [16], the authors treat homogeneous Dirichlet boundary conditions for the Poisson problem in the presence of boundary oscillations and they are able to obtain good estimates on the asymptotic expansion of the solution in terms of the parameter $\epsilon$. We also refer to [17] for an interesting application to a climatization problem. The articles mentioned above and most of the references in the literature address linear problems.

When $g \equiv 0$, that is, we have a homogeneous Neumann boundary condition, the results from [4] show that under the conditions on the perturbation of the present paper (actually only condition $(\mathbf{H})$ would be needed), the Neumann boundary condition is also preserved in the limit, that is, we always have that the limit problem is $\Delta u+f(x, u)=0$ in $\Omega_{0}$ with boundary condition $\frac{\partial u}{\partial n}=0$.

In 12 the authors study linear and nonlinear problems but with a linear Robin boundary conditions of the type $\frac{\partial u}{\partial n}+\beta_{0} u=0$ with $\beta_{0}>0$. That is, they assume $g(x, u)=\beta_{0} u$. They consider different classes of domain perturbations and the third type they study (Theorem 4.4 in [12]) is related to our present work. They show that the limit behaves like in the present paper, that is, the limiting boundary condition is of the type $\frac{\partial u}{\partial n}+\beta_{0} \gamma u=0$ and $\gamma$ is related, as we mentioned above, to the limit of the relative surface measure of $\partial \Omega_{\epsilon}$ with respect to $\partial \Omega$. With the techniques of [12] it is not possible to treat the situation of a general nonlinear boundary condition of the type $\frac{\partial u}{\partial n}+g(x, u)=0$. This is mainly due because with the hypotheses of the domain perturbation from [12 they need to use an inequality due to Maz'ja, (see [15] and also Theorem 2.2 of [12]) which is only applicable when the boundary condition is dissipative, like in the case $\beta_{0}>0$. In our case, we have a little more restrictive perturbations than their third case, but we allow more general boundary conditions, in particular nonlinear ones with nondefined sign nor dissipative properties. Moreover, we also obtain the convergence in stronger norms, like $H^{1}$ and Hölder norms and we also are able to show a uniqueness result, as we mentioned above.

In a different context although related to this article and to [3], the work [10] studies how the non slip condition of the type $u \cdot n=0$ of a fluid passing through a wall with rugosity converges to the condition $u=0$ as the rugosity becomes finer and finer.

This paper is organized as follow: in Section 2, we define the domain perturbation we will consider and state our main results (Theorem [2.4 and Theorem 2.6). In Section [3, we make the considerations about the equations and develop the main functional techniques we will use to prove the results. In Section 4 we consider some important technical results and in Section 5 we prove the main results.

\section{Setting of the problem and main results}

We consider a family of smooth, bounded domains $\Omega_{\epsilon} \subset \mathbb{R}^{N}, N \geq 2$, for $0 \leq \epsilon \leq \epsilon_{0}$, for some $\epsilon_{0}>0$ fixed and we regard $\Omega_{\epsilon}$ as a perturbation of the fixed domain $\Omega \equiv \Omega_{0}$. We consider 
the following condition on the domain

(H) i) for all $K \subset \Omega, K$ compact, there exists $\epsilon(K)>0$ such that $K \subset \Omega_{\epsilon}$ for $0<\epsilon<\epsilon(K)$.

ii) There exists a finite open cover $\left\{U_{i}\right\}_{i=0}^{m}$ of $\Omega$ such that $\bar{U}_{0} \subset \Omega, \partial \Omega \subset \cup_{i=1}^{m} U_{i}$ and for each $i=1, \ldots, m$, there exists a Lipschitz diffeomorphism $\Phi_{i}: Q_{N} \rightarrow U_{i}$, where $Q_{N}=(-1,1)^{N} \subset \mathbb{R}^{N}$, such that

$$
\begin{gathered}
\Phi_{i}\left(Q_{N-1} \times(-1,0)\right)=U_{i} \cap \Omega, \\
\Phi_{i}\left(Q_{N-1} \times\{0\}\right)=U_{i} \cap \partial \Omega .
\end{gathered}
$$

Moreover, we assume that $\bar{\Omega}_{\epsilon} \subset \cup_{i=0}^{m} U_{i} \equiv U$ and for each $i=1, \ldots, m$ there exists Lipschitz functions $\rho_{i, \epsilon}: Q_{N-1} \rightarrow(-1,1)$ such that $\Phi_{i}^{-1}\left(U_{i} \cap \partial \Omega_{\epsilon}\right)$ is the graph of $\rho_{i, \epsilon}$. This means $U_{i} \cap \partial \Omega_{\epsilon}=\Phi_{i}\left(\left\{\left(x^{\prime}, \rho_{i, \epsilon}\left(x^{\prime}\right)\right), x^{\prime} \in Q_{N-1}\right\}\right)$, where we denote $\left(x_{1}, \ldots, x_{N-1}\right)$ by $x^{\prime}$. We assume that $\rho_{i, \epsilon} \rightarrow 0$, when $\epsilon \rightarrow 0, i=1, \ldots, m$, uniformly in $Q_{N-1}$.

Note that if $\Omega \subset \Omega_{\epsilon}$, then condition (H) i) is satisfied.

We consider the following mappings:

$$
T_{i, \epsilon}: Q_{N} \rightarrow Q_{N}
$$

defined by

$$
T_{i, \epsilon}\left(x^{\prime}, s\right)= \begin{cases}\left(x^{\prime}, s+s \rho_{i, \epsilon}\left(x^{\prime}\right)+\rho_{i, \epsilon}\left(x^{\prime}\right)\right), & \text { for } s \in(-1,0) \\ \left(x^{\prime}, s-s \rho_{i, \epsilon}\left(x^{\prime}\right)+\rho_{i, \epsilon}\left(x^{\prime}\right)\right), & \text { for } s \in[0,1) .\end{cases}
$$

Also,

$$
\Phi_{i, \epsilon}:=\Phi_{i} \circ T_{i, \epsilon}: Q_{N} \rightarrow U_{i}
$$

and we also denote by

$$
\begin{aligned}
\phi_{i, \epsilon}: Q_{N-1} & \rightarrow U_{i} \cap \Omega_{\epsilon} \\
x^{\prime} & \rightarrow \Phi_{i, \epsilon}\left(x^{\prime}, 0\right)
\end{aligned}
$$

and

$$
\begin{aligned}
\phi_{i, 0}: Q_{N-1} & \rightarrow U_{i} \cap \Omega \\
x^{\prime} & \rightarrow \Phi_{i}\left(x^{\prime}, 0\right)
\end{aligned}
$$

Notice that $\phi_{i, \epsilon}$ and $\phi_{i}$ are local parameterization of $\partial \Omega_{\epsilon}$ and $\partial \Omega$, respectively. Furthermore, observe that all the maps above are Lipschitz.

In the present paper, we consider the following hypothesis on the deformation of $\partial \Omega_{\epsilon}$ :

(F) i) $\left\|\nabla \rho_{i, \epsilon}\right\|_{L^{\infty}} \leq C$, with $C$ independent of $\epsilon, i=1, \ldots, m$, and

ii) For each $i=1, \ldots, m$, there exists a function $\gamma_{i} \in L^{\infty}\left(Q_{N-1}\right)$ such that

$$
J \phi_{i, \epsilon} \equiv\left|\frac{\partial \phi_{i, \epsilon}}{\partial x_{1}} \wedge \ldots \wedge \frac{\partial \phi_{i, \epsilon}}{\partial x_{N-1}}\right| \stackrel{\epsilon \rightarrow 0}{\longrightarrow} \gamma_{i}, \quad w-L^{1}\left(Q_{N-1}\right)
$$




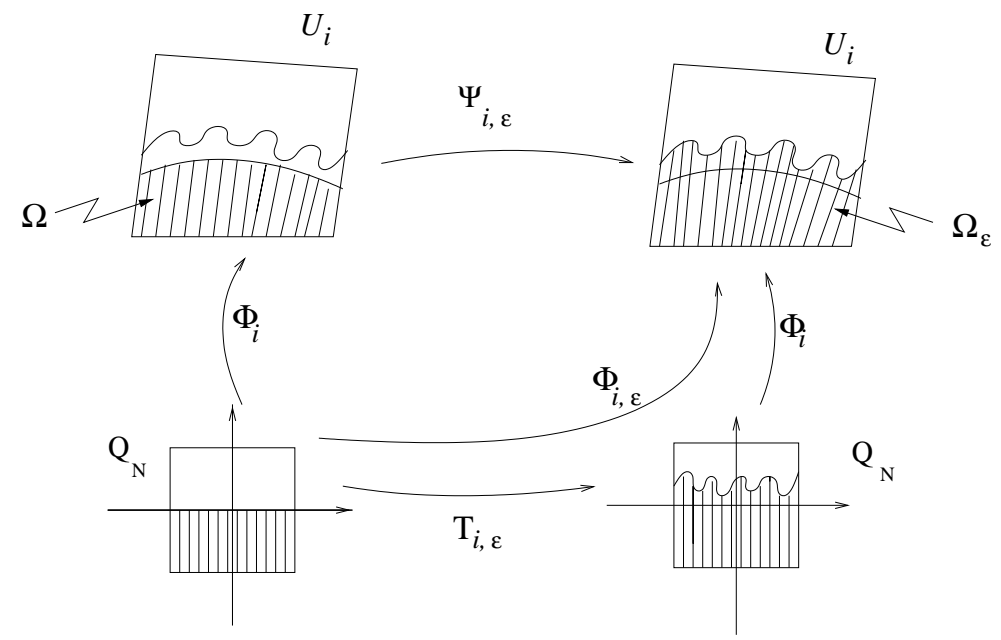

Notice that, by definition,

$$
J \phi_{i, \epsilon}=\sqrt{\sum_{j=1}^{N}\left(\operatorname{det}\left(\operatorname{Jac} \phi_{i, \epsilon}\right)_{j}\right)^{2}}
$$

and $\left(\operatorname{Jac} \phi_{i, \epsilon}\right)_{j}$ is the jacobian matrix without the $j$-th row.

Remark 2.1. Observe that from $(\mathbf{F}) i)$ we get that $J \phi_{i, \epsilon}$ is a bounded sequence in $L^{\infty}\left(Q_{N-1}\right)$. From this and with the aid of (2.3), we obtain that $J \phi_{i, \epsilon} \rightarrow \gamma_{i}, w-L^{q}\left(Q_{N-1}\right)$ for all $1 \leq q<\infty$.

This assertion follows from the following observation: if $U$ is a bounded domain and if $f_{\epsilon} \in L^{\infty}(U), f \in L^{\infty}(U)$ satisfy that $f_{\epsilon} \rightarrow f w-L^{1}(U)$ and $\left\|f_{\epsilon}\right\|_{L^{\infty}(U)} \leq K$, then $f_{\epsilon} \rightarrow f$ $w-L^{q}(U)$, for all $1 \leq q<\infty$. In fact, let $1<p<\infty$ and $\psi \in L^{p}(U)$, then, by density of $C_{0}^{\infty}(U)$ in $L^{p}(U)$, given $\beta>0$ there exists $\psi_{\beta} \in C_{0}^{\infty}(S)$ such that $\left\|\psi-\psi_{\beta}\right\|_{L^{p}(U)} \leq \beta$. Then,

$$
\left|\int_{U}\left(f_{\epsilon}-f\right) \psi\right| \leq \int_{U}\left|f_{\epsilon}-f\right|\left|\psi-\psi_{\beta}\right|+\left|\int_{U}\left(f_{\epsilon}-f\right) \psi_{\beta}\right| .
$$

Using the fact $\left\|f_{\epsilon}\right\|_{L^{\infty}(U)} \leq K$, we get

$$
\int_{U}\left|f_{\epsilon}-f\right|\left|\psi-\psi_{\beta}\right| \leq 2 K|U|^{\frac{1}{p^{\prime}}} \beta .
$$

Since $f_{\epsilon}$ converges to $f$ weakly in $L^{1}(U)$, then $\left|\int_{U}\left(f_{\epsilon}-f\right) \psi_{\beta}\right|$ goes to zero when $\epsilon \rightarrow 0$ Therefore, $\lim _{\epsilon \rightarrow 0}\left|\int_{U}\left(f_{\epsilon}-f\right) \psi\right|=0$, for all $\psi \in L^{p}(U)$, that means $f_{\epsilon} \rightarrow f w-L^{q}(U)$ for $1 \leq q<\infty$.

Now, we define a function $\gamma: \partial \Omega \rightarrow \mathbb{R}$ which will measure the limit of the deformation of $\partial \Omega_{\epsilon}$ relatively to $\partial \Omega$. 
Definition 2.2. We define the function $\gamma$ as

$$
\gamma(x)=\frac{\gamma_{i}(y)}{J \Phi_{i}(y)}, \text { where } y=\Phi_{i}^{-1}(x) \in Q_{N-1}, \text { and } x \in U_{i} \cap \partial \Omega
$$

We observe that the function $\gamma_{i}$ is defined using the sequence $\phi_{i, \epsilon}$. In consequence, $\gamma$ could depend on the choice of $\phi_{i, \epsilon}$ and also on the choice of the charts $U_{i}$ and the maps $\Phi_{i}$. We will prove below that $\gamma$ is well defined and unique for the family $\Omega_{\epsilon}$ and $\Omega$.

Example 2.3. We would like to consider a basic example where we can calculate explicitly the function $\gamma$. Let us assume $N=2$ and let $\Omega_{0} \cap Q_{2}=\left\{\left(x_{1}, x_{2}\right):-1<x_{2}<0\right\}$, so that $\partial \Omega_{0} \cap Q_{2}=\left\{\left(x_{1}, 0\right):-1 \leq x_{1} \leq 1\right\}$. Assume also that $\partial \Omega_{\epsilon} \cap Q_{2}=\left\{\left(x_{1}, \rho_{\epsilon}\left(x_{1}\right)\right):-1<\right.$ $\left.x_{1}<1\right\}$ and $\rho_{\epsilon}\left(x_{1}\right)=\epsilon \sin \left(x_{1} / \epsilon^{\alpha}\right) \varphi\left(x_{1}\right)$, for some smooth function $\varphi$ of compact support in $(-1,1)$ and with $\alpha>0$.

If $0<\alpha \leq 1$, then both hypotheses $(\mathbf{H})$ and $(\mathbf{F})$ are satisfied. Moreover, if $\alpha<1$ we have $\gamma \equiv 1$ and if $\alpha=1$, we have $\gamma(x)=\frac{1}{2 \pi} \int_{0}^{2 \pi} \sqrt{1+(\varphi(x) \cos (z))^{2}} d z$

With respect to the equations, we will be interested in studying the behavior of the solutions of the elliptic equation (1.1) where, as we mentioned in the introduction, the nonlinearities $f: U \times \mathbb{R} \rightarrow \mathbb{R}, g: U \times \mathbb{R} \rightarrow \mathbb{R}$ are continuous in both variables and $C^{2}$ in the second one and $U$ is a bounded domain containing $\bar{\Omega}_{\epsilon}$, for all $0 \leq \epsilon \leq \epsilon_{0}$.

For $0<\epsilon \leq \epsilon_{0}$, We will denote by $\mathcal{E}_{\epsilon}=\left\{u_{\epsilon} \in H^{1}\left(\Omega_{\epsilon}\right): u_{\epsilon}\right.$ is a solution of (1.1) $\}$, $\mathcal{E}_{\epsilon, R}=\left\{u_{\epsilon} \in \mathcal{E}_{\epsilon}:\left\|u_{\epsilon}\right\|_{L^{\infty}\left(\Omega_{\epsilon}\right)} \leq R\right\}$ and $\mathcal{E}_{\epsilon, R^{-}}=\left\{u_{\epsilon} \in \mathcal{E}_{\epsilon}:\left\|u_{\epsilon}\right\|_{L^{\infty}\left(\Omega_{\epsilon}\right)}<R\right\}$

Since we will need to compare functions defined in $\Omega_{\epsilon}$ with functions defined in the unperturbed domain $\Omega_{0}$, we consider the operator $E_{\epsilon}: H^{1}(\Omega) \rightarrow H^{1}\left(\Omega_{\epsilon}\right)$, which is defined as $E_{\epsilon}=R_{\epsilon} \circ E$, where $E: H^{1}(\Omega) \rightarrow H^{1}\left(\mathbb{R}^{N}\right)$ is an extension operator constructed in the usual way and $R_{\epsilon}$ is the restriction operator from functions defined in $\mathbb{R}^{N}$ to functions defined in $\Omega_{\epsilon}$. Observe that we also have $E_{\epsilon}: L^{p}(\Omega) \rightarrow L^{p}\left(\Omega_{\epsilon}\right), E_{\epsilon}: W^{1, p}(\Omega) \rightarrow W^{1, p}\left(\Omega_{\epsilon}\right)$, for all $1 \leq p \leq \infty, E_{\epsilon}: C^{\alpha}(\bar{\Omega}) \rightarrow C^{\alpha}\left(\bar{\Omega}_{\epsilon}\right)$, for $0 \leq \alpha<1$ and that in each case we have $\left\|E_{\epsilon} u\right\|_{X_{\epsilon}} \rightarrow\|u\|_{X_{0}}$ where $X_{\epsilon}=H^{1}\left(\Omega_{\epsilon}\right), L^{p}\left(\Omega_{\epsilon}\right), W^{1, p}\left(\Omega_{\epsilon}\right), C^{\alpha}\left(\bar{\Omega}_{\epsilon}\right), \epsilon \geq 0$.

Our main results are stated in the following theorems.

Theorem 2.4. Assume $(\mathbf{H})$ and $(\mathbf{F})$ are satisfied. Let $u_{\epsilon}^{*}, 0<\epsilon \leq \epsilon_{0}$, be a family of solutions of problem (1.1) satisfying $\left\|u_{\epsilon}^{*}\right\|_{L^{\infty}\left(\Omega_{\epsilon}\right)} \leq R$ for some constant $R>0$ independent of $\epsilon$, that is $u_{\epsilon}^{*} \in \mathcal{E}_{\epsilon, R}$. We have the following:

i) There exists a subsequence, still denoted by $u_{\epsilon}^{*}$, and a function $u_{0}^{*} \in \mathcal{E}_{0, R}$, that is $u_{0}^{*} \in H^{1}(\Omega)$ with $\left\|u_{0}^{*}\right\|_{L^{\infty}(\Omega)} \leq R$, solution of problem (1.2) with the property that $\left\|u_{\epsilon}^{*}-E_{\epsilon} u_{0}^{*}\right\|_{H^{1}\left(\Omega_{\epsilon}\right)}+$ $\left\|u_{\epsilon}^{*}-E_{\epsilon} u_{0}^{*}\right\|_{C^{\beta}\left(\bar{\Omega}_{\epsilon}\right)} \rightarrow 0$ as $\epsilon \rightarrow 0$, for some $0<\beta \leq 1$.

ii) If the equilibrium point $u_{0}^{*}$ is hyperbolic, in the sense that $\lambda=0$ is not an eigenvalue of the linearized problem of (1.2) around $u_{0}^{*}$ (see problem (2.7) below), then, there exists $\delta>0$ small such that problem (1.1) has one and only one solution $u_{\epsilon}^{*} \in \mathcal{E}_{\epsilon, R^{-}}$satisfying $\left\|u_{\epsilon}^{*}-E_{\epsilon} u_{0}^{*}\right\|_{H^{1}\left(\Omega_{\epsilon}\right)} \leq \delta$ for $\epsilon$ small enough. 
Remark 2.5. Since in Theorem 2.4 we are concerned with solutions satisfying a uniform bound of the type $\left\|u_{\epsilon}\right\|_{L^{\infty}\left(\Omega_{\epsilon}\right)} \leq R$, we may modify the nonlinearities $f$ and $g$ outside the region $|u| \leq R$ without modifying any of these solutions. Hence, we may perform a cut-off in the nonlinearities $f$ and $g$ in such a way that

$$
\begin{aligned}
& |f(x, u)|+\left|\partial_{u} f(x, u)\right|+\left|\partial_{u u} f(x, u)\right| \leq C, \quad x \in U, \quad u \in \mathbb{R} \\
& |g(x, u)|+\left|\partial_{u} g(x, u)\right|+\left|\partial_{u u} g(x, u)\right| \leq C, \quad x \in U, \quad u \in \mathbb{R}
\end{aligned}
$$

Also, we will be able to prove the spectral convergence of the linearizations around the equilibrium points. Observe that if $u_{\epsilon}^{*}$ is a solution of (1.1) then, the spectra of the linearization of (1.1) around $u_{\epsilon}^{*}$ is given by the eigenvalue problem

$$
\begin{cases}-\Delta w+w-\partial_{u} f\left(x, u_{\epsilon}^{*}\right) w=\lambda w & \text { in } \Omega_{\epsilon}, \\ \frac{\partial w}{\partial n}+\partial_{u} g\left(x, u_{\epsilon}^{*}\right) w=0 & \text { on } \partial \Omega_{\epsilon} .\end{cases}
$$

Similarly, if $u_{0}^{*}$ is a solution of (1.2), then the spectra of its linearization is given by the eigenvalue problem

$$
\begin{cases}-\Delta w+w-\partial_{u} f\left(x, u_{0}^{*}\right) w=\lambda w & \text { in } \Omega, \\ \frac{\partial w}{\partial n}+\gamma \partial_{u} g\left(x, u_{0}^{*}\right) w=0 & \text { on } \partial \Omega .\end{cases}
$$

Notice that both problems, (2.6) and (2.7), are selfadjoint and of compact resolvent. Hence, the eigenvalues of (2.6) are given by a sequence $\left\{\lambda_{n}^{\epsilon}\right\}_{n=1}^{\infty}$, ordered and counting their multiplicity, with $\lambda_{n}^{\epsilon} \rightarrow+\infty$ as $n \rightarrow+\infty$. Similarly the eigenvalues of (2.7) are also given by a sequence $\left\{\lambda_{n}^{0}\right\}_{n=1}^{\infty}$ with $\lambda_{n}^{0} \rightarrow+\infty$.

Theorem 2.6. With the notations above and in the conditions of Theorem 2.4, if $u_{\epsilon}^{*} \in \mathcal{E}_{\epsilon, R}$ for $0 \leq \epsilon \leq \epsilon_{0}$ and $\left\|u_{\epsilon}^{*}-E_{\epsilon} u_{0}^{*}\right\|_{H^{1}\left(\Omega_{\epsilon}\right)} \rightarrow 0$, then the eigenvalues and eigenfunctions of (2.6) converge to the eigenvalues and eigenfunctions of (2.7). That is, for each fixed $n \in \mathbb{N}$, $\lambda_{n}^{\epsilon} \rightarrow \lambda_{n}^{0}$, as $\epsilon \rightarrow 0$. Moreover, if we denote by $\left\{\varphi_{n}^{\epsilon}\right\}_{n=1}^{\infty}$ a set of orthonormal eigenfunctions associated to $\left\{\lambda_{n}^{\epsilon}\right\}_{n=1}^{\infty}$, then for each sequence $\epsilon_{k} \rightarrow 0$ there is another subsequence, that we still denote by $\epsilon_{k}$, and a set of orthonormal eigenfunctions $\left\{\varphi_{n}^{0}\right\}_{n=1}^{\infty}$ associated to $\left\{\lambda_{n}^{0}\right\}_{n=1}^{\infty}$, such that, for all $n \in \mathbb{N}$, we have $\left\|\varphi_{n}^{\epsilon_{k}}-E_{\epsilon} \varphi_{n}^{0}\right\|_{H^{1}\left(\Omega_{\epsilon_{k}}\right)} \rightarrow 0$ as $\epsilon_{k} \rightarrow 0$.

Remark 2.7. i) The convergence of the eigenfunctions in Theorem [2.6 can be expressed in terms of the convergence of the eigenspaces, in the sense that if $n \in \mathbb{N}$ is such that $\lambda_{n}^{0}<\lambda_{n+1}^{0}$, and if we denote by $W_{n}^{\epsilon}$ the linear subspace spanned by the first $n$ eigenfunctions, that is $W_{n}^{\epsilon}=\left[\varphi_{1}^{\epsilon}, \ldots, \varphi_{n}^{\epsilon}\right] \subset H^{1}\left(\Omega_{\epsilon}\right) 0 \leq \epsilon \leq \epsilon_{0}$, then the distance between $W_{n}^{\epsilon}$ and $E_{\epsilon} W_{n}^{0}$ approaches 0 as $\epsilon \rightarrow 0$.

Notice also that if $\lambda_{n}^{0}$ is a simple eigenvalue, then $\lambda_{n}^{\epsilon}$ is also simple for $\epsilon$ small enough and, via subsequences, we always have that $\varphi_{n}^{\epsilon_{k}} \rightarrow \varphi_{n}^{0}$ or $\varphi_{n}^{\epsilon_{k}} \rightarrow-\varphi_{n}^{0}$ as $\epsilon \rightarrow 0$.

ii) Theorem 2.6 has important implications for understanding the behavior of the dynamics of the associated parabolic equations. If we regard (1.1) and (1.2) as the stationary equations 
of the parabolic evolutionary equations

$$
\begin{cases}u_{t}-\Delta u+u=f(x, u) & \text { in } \Omega_{\epsilon} \\ \frac{\partial u}{\partial n}+g(x, u)=0 & \text { on } \partial \Omega_{\epsilon} \\ u(x, 0)=u_{0}(x) \in H^{1}\left(\Omega_{\epsilon}\right) & \end{cases}
$$

and

$$
\left\{\begin{array}{l}
u_{t}-\Delta u+u=f(x, u) \text { in } \Omega \\
\frac{\partial u}{\partial n}+\gamma g(x, u)=0 \text { in } \partial \Omega \\
u(x, 0)=u_{0}(x) \in H^{1}(\Omega)
\end{array}\right.
$$

respectively, then, among other things, Theorem 2.6 is saying that if $u_{0}^{*}$ is a linearly asymptotically stable equilibrium point of (2.9) and $u_{\epsilon}^{*}$ are the equilibria obtained in Theorem 2.4. then $u_{\epsilon}^{*}$ are also linearly asymptotically stable. Also, if $u_{0}^{*}$ is a hyperbolic equilibrium then, the linear unstable manifold associated to $u_{\epsilon}^{*}$ converge to the linear unstable manifold associated to $u_{0}^{*}$.

\section{Solutions as fixed points and E-convergence}

The solutions of (1.1) and (1.2) will be obtained as fixed points of appropriate nonlinear maps defined in the spaces $H^{1}\left(\Omega_{\epsilon}\right)$ and $H^{1}(\Omega)$, respectively. This maps are constructed in Subsection 3.1. Moreover, since these maps are defined in different spaces, we will need a tool to compare functions, operators, etc., which are defined in different spaces. Moreover, we will need a precise definition of weak and strong convergence of functions defined in different spaces and to develop certain analytical results to be able to deal with this situation. The appropriate notion for this is the concept of $E$-convergence and a key ingredient for this will be the use of the extension operator $E_{\epsilon}: H^{1}(\Omega) \rightarrow H^{1}\left(\Omega_{\epsilon}\right)$ defined in Section 2 . The definition and basic properties of $E$-convergence will be done in Subsection 3.2 .

\subsection{Fixed points}

For $0<\epsilon \leq \epsilon_{0}$, consider the linear operator $A_{\epsilon}: D\left(A_{\epsilon}\right) \subset L^{2}\left(\Omega_{\epsilon}\right) \rightarrow L^{2}\left(\Omega_{\epsilon}\right)$ defined by $A_{\epsilon} u_{\epsilon}=-\Delta u_{\epsilon}+u_{\epsilon}$ with domain $D\left(A_{\epsilon}\right)=\left\{u_{\epsilon} \in H^{2}\left(\Omega_{\epsilon}\right): \frac{\partial u_{\epsilon}}{\partial n}=0\right\}$. Let us denote by $E_{\epsilon}^{0}=L^{2}\left(\Omega_{\epsilon}\right), E_{\epsilon}^{1}=D\left(A_{\epsilon}\right)$ and consider the scale of Hilbert spaces $\left\{\left(E_{\epsilon}^{\alpha}, A_{\epsilon}^{\alpha}\right), \alpha \in R\right\}$ constructed by complex interpolation, see [1, which coincide, since we are in a Hilbert setting, with the standard fractional power spaces of the operator $A_{\epsilon}$. Also, $E_{\epsilon}^{\alpha} \hookrightarrow H_{\epsilon}^{2 \alpha}$. This scale can also be extended to spaces of negative exponents by taking $E_{\epsilon}^{-\alpha}=\left(E_{\epsilon}^{\alpha}\right)^{\prime}$, for $\alpha>0$, and $E_{\epsilon}^{-\frac{1}{2}}=H^{-1}\left(\Omega_{\epsilon}\right)$. Considering the realizations of $A_{\epsilon}$ in this scale, the operator $A_{\epsilon,-\frac{1}{2}} \in L\left(E_{\epsilon}^{\frac{1}{2}}, E_{\epsilon}^{-\frac{1}{2}}\right)$, is given by

$$
\left\langle A_{\epsilon,-\frac{1}{2}} u_{\epsilon}, \phi_{\epsilon}\right\rangle=\int_{\Omega_{\epsilon}} \nabla u_{\epsilon} \nabla \phi_{\epsilon}+u_{\epsilon} \phi_{\epsilon},
$$


for $\phi_{\epsilon} \in H^{1}\left(\Omega_{\epsilon}\right)$. With some abuse of notation we will identify all different realizations of this operators and we will write them all as $A_{\epsilon}$.

With this considerations, we write (1.1) in an abstract form as

$$
A_{\epsilon} u_{\epsilon}=h_{\epsilon}\left(u_{\epsilon}\right)
$$

where $h_{\epsilon}: H^{1}\left(\Omega_{\epsilon}\right) \rightarrow H^{-\alpha}\left(\Omega_{\epsilon}\right)$ with $\frac{1}{2}<\alpha<1$, defined by

$$
\left\langle h_{\epsilon}\left(u_{\epsilon}\right), \phi_{\epsilon}\right\rangle=\int_{\Omega_{\epsilon}} f\left(x, u_{\epsilon}\right) \phi_{\epsilon}-\int_{\partial \Omega_{\epsilon}} g\left(x, u_{\epsilon}\right) \phi_{\epsilon}, \quad \phi_{\epsilon} \in H^{\alpha}\left(\Omega_{\epsilon}\right) .
$$

In particular, $u_{\epsilon}$ is a solution of (1.1) if and only if $u_{\epsilon}$ satisfies $u_{\epsilon}=A_{\epsilon}^{-1} h_{\epsilon}\left(u_{\epsilon}\right)$, that is, $u_{\epsilon}$ is a fixed point of the nonlinear map $A_{\epsilon}^{-1} \circ h_{\epsilon}: H^{1}\left(\Omega_{\epsilon}\right) \rightarrow H^{1}\left(\Omega_{\epsilon}\right)$.

In a very similar way, the solutions of the limiting equation (1.2) can be written as fixed points of the map $A_{0}^{-1} \circ h: H^{1}(\Omega) \rightarrow H^{1}(\Omega)$ where $A_{0}: D\left(A_{0}\right) \subset L^{2}(\Omega) \rightarrow L^{2}(\Omega)$ the linear operator defined by $A_{0} u=-\Delta u+u$, with domain $D\left(A_{0}\right)=\left\{u \in H^{2}(\Omega): u=0\right.$ in $\left.\partial \Omega\right\}$ and $h: H^{1}(\Omega) \rightarrow H^{-\alpha}(\Omega)$, with $\frac{1}{2}<\alpha<1$, defined by

$$
\langle h(u), \phi\rangle=\int_{\Omega} f(x, u) \phi-\int_{\partial \Omega} \gamma g(x, u) \phi, \quad \phi \in H^{\alpha}(\Omega) .
$$

\section{$3.2 \quad E$-convergence}

In this subsection we are going to develop the basic tools which will be used to compare the solutions of two problems defined in different spaces. We will not develop the complete theory and will only mention the results that apply directly to our case. We refer to [9] for the general theory and to [5] for a concrete application to a domain perturbation problem, different from the present one.

In our setting we will have a family of Hilbert spaces, $H_{\epsilon}, 0<\epsilon \leq \epsilon_{0}$ and we will also have a "limiting" Hilbert space $H$. We denote by $(\cdot, \cdot)_{\epsilon}$ the inner product in $H_{\epsilon}$, and by $(\cdot, \cdot)$ the inner product in $H$. For instance, we may consider $H_{\epsilon}=H^{1}\left(\Omega_{\epsilon}\right)$ and $H=H^{1}(\Omega)$.

We consider $E_{\epsilon}: H \rightarrow H_{\epsilon}$ a family of linear continuous operators, such that

$$
\left\|E_{\epsilon} u\right\|_{H_{\epsilon}} \rightarrow\|u\|_{H}, \text { when } \epsilon \rightarrow 0
$$

Our basic notion of convergence and weak convergence is stated in the following definitions.

Definition 3.1. A sequence of elements $\left\{u_{\epsilon}\right\}, u_{\epsilon} \in H_{\epsilon}, \epsilon>0$, is said to be E-convergent to $u \in H$ if $\left\|u_{\epsilon}-E_{\epsilon} u\right\|_{H_{\epsilon}} \rightarrow 0$ as $\epsilon \rightarrow 0$. We write this as $u_{\epsilon} \stackrel{E}{\longrightarrow} u$.

Definition 3.2. A sequence of elements $\left\{u_{\epsilon}\right\}, u_{\epsilon} \in H_{\epsilon}, \epsilon>0$, is said to be E-weakly convergent to $u \in H$ if for any sequence $w_{\epsilon}$ E-convergent to $w$ implies $\left(w_{\epsilon}, u_{\epsilon}\right)_{\epsilon} \rightarrow(w, u)$, when $\epsilon \rightarrow 0$. We denote by $u_{\epsilon} \stackrel{E}{\longrightarrow} u$. 
Some important properties of this convergence are stated in the following results.

Proposition 3.3. If $\left\|u_{\epsilon}\right\|_{H_{\epsilon}} \leq K, u \in H$ and for all $w \in H,\left(E_{\epsilon} w, u_{\epsilon}\right)_{\epsilon} \rightarrow(w, u)$ when $\epsilon \rightarrow 0$, then $u_{\epsilon} \stackrel{E}{\longrightarrow} u$.

Proof. Let $w_{\epsilon} \stackrel{E}{\longrightarrow} w$. Then, $\left(w_{\epsilon}, u_{\epsilon}\right)_{\epsilon}=\left(w_{\epsilon}-E_{\epsilon} w, u_{\epsilon}\right)_{\epsilon}-\left(E_{\epsilon} w, u_{\epsilon}\right)_{\epsilon} \rightarrow(w, u)$.

Proposition 3.4. If $u_{\epsilon} \stackrel{E}{\longrightarrow} u$ and $\limsup _{\epsilon \rightarrow 0}\left\|u_{\epsilon}\right\|_{\epsilon} \leq\|u\|$ then $u_{\epsilon} \stackrel{E}{\longrightarrow} u$.

Proof. Since $0 \leq\left\|u_{\epsilon}-E_{\epsilon} u\right\|_{H_{\epsilon}}^{2}=\left\|u_{\epsilon}\right\|_{H_{\epsilon}}^{2}-2\left(u_{\epsilon}, E_{\epsilon} u\right)_{\epsilon}+\left\|E_{\epsilon} u\right\|_{H_{\epsilon}}^{2}$ we get the result.

We will need a notion of compactness and of convergence of operators which are defined in different spaces. These notions are stated in the following.

Definition 3.5. A sequence of elements $\left\{u_{n}\right\}, u_{n} \in H_{\epsilon_{n}}, n \in \mathbb{N}$, is said to be E-precompact if for any subsequence $\left\{u_{n^{\prime}}\right\}$ there exist a subsequence $\left\{u_{n^{\prime \prime}}\right\}$ and $u \in H$ such that $u_{n^{\prime \prime}} \stackrel{E}{\longrightarrow} u$, as $n^{\prime \prime} \rightarrow \infty$. A family $\left\{u_{\epsilon}\right\}, \epsilon \in(0,1]$ is said pre-compact if each sequence $\left\{u_{\epsilon_{n}}\right\}$, with $\epsilon_{n} \rightarrow 0$, is pre-compact.

Definition 3.6. We say that a family of operators $T_{\epsilon}: H_{\epsilon} \rightarrow H_{\epsilon}, \epsilon \in(0,1]$, E-converges to $T: H \rightarrow H$ as $\epsilon \rightarrow 0$, if $T_{\epsilon} u_{\epsilon} \stackrel{E}{\longrightarrow} T u$, whenever $u_{\epsilon} \stackrel{E}{\longrightarrow} u \in H$. We denote this by $T_{\epsilon} \stackrel{E E}{\longrightarrow} T$.

Finally, we have the following important notion on compact convergence of operators.

Definition 3.7. We say that a family of compact operators $T_{\epsilon}: H_{\epsilon} \rightarrow H_{\epsilon}, \epsilon \in(0,1]$ converges compactly to a compact $T: H \rightarrow H$ if for any family $u_{\epsilon}$ with $\left\|u_{\epsilon}\right\|_{\epsilon}$ bounded, the family $\left\{T_{\epsilon} u_{\epsilon}\right\}$ is E-precompact and $T_{\epsilon} \stackrel{E E}{\longrightarrow} T$. We write $T_{\epsilon} \stackrel{C C}{\longrightarrow} T$.

An important result on convergence of fixed points is the following:

Theorem 3.8. Let $T_{\epsilon}: H_{\epsilon} \rightarrow H_{\epsilon}$ be a family of compact operators such that $T_{\epsilon} \stackrel{C C}{\longrightarrow} T$. Let $u_{\epsilon}$ be a fixed point of $T_{\epsilon}$ such that $\left\|u_{\epsilon}\right\|_{H_{\epsilon}}$ is uniformly bounded. Then, there exists a subsequence $u_{\epsilon_{k}}$ and $u \in H$ with $u=T u$ such that $u_{\epsilon_{k}} \stackrel{E}{\longrightarrow} u$.

Proof. Since $\left\|u_{\epsilon}\right\|_{H_{\epsilon}}$ is uniformly bounded, by Definition [3.7. $T_{\epsilon} u_{\epsilon}$ is E-precompact. Thus, there is a sequence $u_{\epsilon_{k}}$, and an element $u \in H$ such that $T_{\epsilon_{k}} u_{\epsilon_{k}} \stackrel{E}{\longrightarrow} u$. Hence, $u_{\epsilon_{k}}=T_{\epsilon_{k}} u_{\epsilon_{k}} \stackrel{E}{\longrightarrow} u$ and by compact convergence, $T_{\epsilon_{k}} u_{\epsilon_{k}} \stackrel{E}{\longrightarrow} T u$. That is, $u=T u$.

In the case where the operators involved are linear, we have some important results.

Lemma 3.9. Assume that $T_{\epsilon} \in \mathcal{L}\left(H_{\epsilon}\right)$ converges compactly to $T \in \mathcal{L}(H)$ as $\epsilon \rightarrow 0$. Then, i) $\left\|T_{\epsilon}\right\|_{\mathcal{L}\left(H_{\epsilon}\right)} \leq C$ for some constant $C$, independent of $\epsilon$.

ii) Assume that $\mathcal{N}(I+T)=\{0\}$ then, there exists an $\epsilon_{0}>0$ and $M>0$ such that

$$
\left\|\left(I+T_{\epsilon}\right)^{-1}\right\|_{\mathcal{L}\left(H_{\epsilon}\right)} \leq M, \quad \forall \epsilon \in\left[0, \epsilon_{0}\right] .
$$


Proof. This result is exactly Lemma 4.7 in [5]. For the sake of completeness and since the proof is short, we include it here.

i) If the norms are not bounded, then we can choose a sequence of $\epsilon_{n} \rightarrow 0$ and $u_{\epsilon_{n}} \in H_{\epsilon_{n}}$ with $\left\|u_{\epsilon_{n}}\right\|_{H_{\epsilon_{n}}}=1$ such that $\left\|T_{\epsilon_{n}} u_{\epsilon_{n}}\right\| \rightarrow+\infty$. But this is in contradiction with the compact convergence of $T_{\epsilon}$ given in Definition 3.7

ii) Since $T_{\epsilon}$ is compact for every $\epsilon \in[0,1]$, the estimate (3.4) is equivalent to say that

$$
\left\|\left(I+T_{\epsilon}\right) u_{\epsilon}\right\|_{H_{\epsilon}} \geqslant \frac{1}{M}, \quad \forall \epsilon \in\left[0, \epsilon_{0}\right] \text { and } \forall u_{\epsilon} \in H_{\epsilon} \text { with }\left\|u_{\epsilon}\right\|=1 .
$$

Suppose that this is not true; that is, suppose that there is a sequence $\left\{u_{n}\right\}$, with $u_{n} \in U_{\epsilon_{n}}$, $\left\|u_{n}\right\|=1$ and $\epsilon_{n} \rightarrow 0$ such that $\left\|\left(I+T_{\epsilon_{n}}\right) u_{n}\right\| \rightarrow 0$. Since $\left\{T_{\epsilon_{n}} u_{n}\right\}$ has a convergent subsequence, which we again denote by $\left\{T_{\epsilon_{n}} u_{n}\right\}$, to $u,\|u\|=1$, then $u_{n}+T_{\epsilon_{n}} u_{n} \rightarrow 0$ and $u_{n} \rightarrow-u$. This implies that $(I+T) u=0$ contradicting our hypothesis.

In many instances, the operators $T_{\epsilon}$ will be inverses of certain differential operators $A_{\epsilon}$. Therefore, let us assume that we have operators $A_{\epsilon}: D\left(A_{\epsilon}\right) \subset H_{\epsilon} \rightarrow H_{\epsilon}$, with well defined inverses and denote by $T_{\epsilon}=A_{\epsilon}^{-1}: H_{\epsilon} \rightarrow H_{\epsilon}$.

One important implication of the compact convergence of linear operators is the convergence of the spectra and of the spectral projections. Since the operators involved are compact, then the spectrum is discrete and the convergence of the spectra will mean the pointwise convergence of the eigenvalues. For the convergence of the spectral projections we need a concept of convergence of linear spaces. Hence, we will say that a family of subspaces $W_{\epsilon} \subset H_{\epsilon} E$-converges to $W_{0} \subset H$ and we will write it as $W_{\epsilon} \stackrel{E}{\longrightarrow} W_{0}$, if $\operatorname{dist}_{H_{\epsilon}}\left(B_{W_{\epsilon}}\right.$, $\left.E_{\epsilon} B_{W_{0}}\right) \rightarrow 0$ as $\epsilon \rightarrow 0$, where $B_{W}$ is the unit ball of the space $W$ and $d_{i s t} t_{H_{\epsilon}}$ is the symmetric Hausdorff distance of two sets in $H_{\epsilon}$.

We can show,

Proposition 3.10. If $A_{\epsilon}: D\left(A_{\epsilon}\right) \subset H_{\epsilon} \rightarrow H_{\epsilon}$ is a closed operator, with compact resolvent and $0 \in \rho\left(A_{\epsilon}\right)$ and $A_{0}: D\left(A_{0}\right) \subset H \rightarrow H$ is also closed, with compact resolvent and $0 \in \rho(A)$, then if $A_{\epsilon}^{-1} \stackrel{C C}{\longrightarrow} A_{0}^{-1}$, then the eigenvalues and eigenfunctions of $A_{\epsilon}$ converge to the eigenvalues and eigenfunctions of $A_{0}$. That is, if $\bar{B}\left(\lambda_{0}, \rho_{0}\right) \subset \mathbb{C}$ lies in the resolvent set of $A_{0}$, then, there exists $\epsilon_{0}=\epsilon_{0}\left(\lambda_{0}, \rho_{0}\right)$, such that the ball is also contained in the resolvent set of $A_{\epsilon}$ for all $0<\epsilon \leq \epsilon_{0}$. Moreover, if $\lambda_{0} \in \sigma\left(A_{0}\right), \bar{B}\left(\lambda_{0}, \rho_{0}\right) \cap \sigma\left(A_{0}\right)=\left\{\lambda_{0}\right\}$ and $W_{0}$ is the generalized eigenspace associated to $\lambda_{0}$, then there exists $\epsilon_{0}=\epsilon_{0}\left(\lambda_{0}, \rho_{0}\right)>$ such that for $0<\epsilon \leq \epsilon_{0}, B\left(\lambda_{0}, \rho_{0}\right) \cap \sigma\left(A_{\epsilon}\right)=\left\{\lambda_{1}^{\epsilon}, \ldots, \lambda_{k(\epsilon)}^{\epsilon}\right\}$ and if $W_{\epsilon}=\operatorname{span}\left\{W_{1}^{\epsilon}, \ldots, W_{k(\epsilon)}^{\epsilon}\right\}$, where $W_{j}^{\epsilon}$ is the generalized eigenspace associated $\lambda_{j}^{\epsilon}$, then $\operatorname{dim}\left(W_{\epsilon}\right)=\operatorname{dim}\left(W_{0}\right)$ and $W_{\epsilon} \stackrel{E}{\longrightarrow} W_{0}$.

Proof. For a proof of this result we refer to Lemma 4.8, Lemma 4.9 and Theorem 4.10 in [5]. 


\section{Some technical results}

In this section we will prove several important technical results that will be needed in the proof of the main result.

We start analyzing extension operators from $\Omega_{\epsilon}$ to $\mathbb{R}^{N}$, Sobolev embeddings and Trace theorems. We will pay special attention to their dependence on the parameter $\epsilon$. As a general remark, observe that $\Omega_{\epsilon} \cap U_{i}$ is a Lipschitz deformation of the fixed domain $Q_{N-1} \times(-1,0)$ and the Lipschitz norm of the transformation is uniformly controlled in $\epsilon$, as $\epsilon \rightarrow 0$, by hypothesis (F) i). This fact will allow us to obtain uniform estimates of constants of Sobolev embeddings and trace theorems.

Let us start with the following useful result.

Lemma 4.1. Consider $S_{\epsilon}$ a family of Lipschitz bounded domains in $\mathbb{R}^{N}$ for $0 \leq \epsilon \leq \epsilon_{0}$. Assume there exists a family of Lipschitz, one-to-one mappings $H_{\epsilon}$ from $S_{0}$ onto $S_{\epsilon}$ such that its inverse $H_{\epsilon}^{-1}$ is Lipschitz, satisfying $\left\|D H_{\epsilon}\right\|_{L^{\infty}\left(S_{0}\right)^{N \times N}} \leq K$ and $\left\|D H_{\epsilon}^{-1}\right\|_{L^{\infty}\left(S_{\epsilon}\right)^{N \times N}} \leq K$, with $K$ independent of $\epsilon$. Then, if we denote by $X(V)=L^{p}(V)$ or $W^{1, p}(V)$ or $C^{\alpha}(V)$, with $1 \leq p \leq \infty$ and $0 \leq \alpha<1$ and $V$ an open set or $\mathbb{R}^{N}$, then, $u \in X\left(S_{\epsilon}\right)$ if and only if $u \circ H_{\epsilon} \in X\left(S_{0}\right)$. Moreover, there exist $C, D$ positive constants independent of $\epsilon$ such that

$$
C\left\|u \circ H_{\epsilon}\right\|_{X\left(S_{0}\right)} \leq\|u\|_{X\left(S_{\epsilon}\right)} \leq D\left\|u \circ H_{\epsilon}\right\|_{X\left(S_{0}\right)} .
$$

Moreover, if $u \in W^{1, p}\left(S_{\epsilon}\right)$,

$$
C\left\|u \circ H_{\epsilon}\right\|_{L^{q}\left(\partial S_{0}\right)} \leq\|u\|_{L^{q}\left(\partial S_{\epsilon}\right)} \leq D\left\|u \circ H_{\epsilon}\right\|_{L^{q}\left(\partial S_{0}\right)}, \quad 1 \leq q \leq \frac{N p-p}{N-p}
$$

Proof. Let us work out first the proof for the case $X=W^{1, p}$. For $\epsilon$ fixed, it is known (see [8] ) that $u \in W^{1, p}\left(S_{\epsilon}\right)$ iff $u \circ H_{\epsilon} \in W^{1, p}\left(S_{0}\right)$. We prove $C\left\|u \circ H_{\epsilon}\right\|_{W^{1, p}\left(S_{0}\right)} \leq\|u\|_{W^{1, p}\left(S_{\epsilon}\right)}$. In fact, since

$$
\frac{\partial}{\partial y_{j}}\left(u \circ H_{\epsilon}\right)(y)=\sum_{l=1}^{N} \frac{\partial u}{\partial x_{l}}\left(H_{\epsilon}(y)\right) \frac{\partial\left(H_{\epsilon}\right)_{l}}{\partial y_{j}}(y)
$$

and using $\left\|D H_{\epsilon}\right\|_{L^{\infty}(S)^{N \times N}} \leq K$ and $\left\|D H_{\epsilon}^{-1}\right\|_{L^{\infty}\left(S_{\epsilon}\right)^{N \times N}} \leq K$, we get

$$
\left\|\left(\left(\frac{\partial u}{\partial x_{l}}\right) \circ H_{\epsilon}\right) \frac{\partial\left(H_{\epsilon}\right)_{l}}{\partial y_{j}}\right\|_{L^{p}(S)} \leq M\left\|\frac{\partial u}{\partial x_{l}}\right\|_{L^{p}\left(S_{\epsilon}\right)}
$$

and

$$
\left\|u \circ H_{\epsilon}\right\|_{L^{p}(S)} \leq M\|u\|_{L^{p}\left(S_{\epsilon}\right)}
$$

then $\left\|u \circ H_{\epsilon}\right\|_{W^{1, p}(S)} \leq \tilde{C}\|u\|_{W^{1, p}\left(S_{\epsilon}\right)}$. The other inequality is obtained similarly.

For the Hölder norm, notice that

$$
\left\|u \circ H_{\epsilon}\right\|_{C^{\beta}\left(S_{0}\right)}=\left\|u \circ H_{\epsilon}\right\|_{L^{\infty}\left(S_{0}\right)}+\sup _{x, y \in S_{0}, x \neq y} \frac{\left|u \circ H_{\epsilon}(x)-u \circ H_{\epsilon}(y)\right|}{|x-y|^{\beta}}
$$




$$
\begin{aligned}
& =\|u\|_{L^{\infty}\left(S_{\epsilon}\right)}+\sup _{x, y \in S_{0}, x \neq y} \frac{\left|u \circ H_{\epsilon}(x)-u \circ H_{\epsilon}(y)\right|}{\left|H_{\epsilon}(x)-H_{\epsilon}(y)\right|^{\beta}} \frac{\left|H_{\epsilon}(x)-H_{\epsilon}(y)\right|^{\beta}}{|x-y|^{\beta}} \\
& \leq\|u\|_{L^{\infty}\left(S_{\epsilon}\right)}+\sup _{z, w \in S_{\epsilon}, z \neq w} \frac{|u(z)-u(w)|}{|z-w|^{\beta}} \sup _{x, y \in S_{\epsilon}, x \neq y} \frac{\left|H_{\epsilon}(x)-H_{\epsilon}(y)\right|^{\beta}}{|x-y|^{\beta}} \\
& \leq\|u\|_{L^{\infty}\left(S_{\epsilon}\right)}+\sup _{x, w \in S_{\epsilon}, z \neq w} \frac{|u(z)-u(w)|}{|z-w|^{\beta}} K^{\beta} \leq \max \left\{1, K^{\beta}\right\}\|u\|_{C^{\beta}\left(S_{\epsilon}\right)} .
\end{aligned}
$$

And the other inequality follows in a similar way. Also, the inequality of the trace is obtained in an analogous way.

Remark 4.2. As an important example of mappings satisfying the hypotheses of Lemma 4.1] we mention the family of maps

$$
\Phi_{i, \epsilon}: Q_{N}^{-} \rightarrow U_{i} \cap \Omega_{\epsilon}
$$

where we denote by $Q_{N}^{-}=Q_{N-1} \times(-1,0) \subset Q_{N}$ and we assume hypotheses $(\mathbf{H})$ and $\left.(\mathbf{F}) i\right)$ are both satisfied.

Now, we show:

Proposition 4.3. Let $\Omega_{\epsilon}$ be a family of domains satisfying conditions $(\mathbf{H})$ and $\left.(\mathbf{F}) i\right)$. Then for each $\epsilon$ and $1 \leq p \leq \infty$, there is a continuous extension operator $P_{\Omega_{\epsilon}}: L^{1}\left(\Omega_{\epsilon}\right) \rightarrow L^{1}\left(\mathbb{R}^{N}\right)$, such that with the notation of $X(V)$ from Lemma 4.1 , then $P_{\Omega_{\epsilon}}$ transforms $X\left(\Omega_{\epsilon}\right)$ into $X\left(\mathbb{R}^{N}\right)$ and

$$
\left\|P_{\Omega_{\epsilon}}\right\|_{\mathcal{L}\left(X\left(\Omega_{\epsilon}\right), X\left(\mathbb{R}^{N}\right)\right)} \leq K, \text { for } 0<\epsilon \leq \epsilon_{0} .
$$

Moreover, the way in which the extension operator $P_{\Omega_{\epsilon}}$ is constructed, we have $P_{\Omega_{\epsilon}} u_{\epsilon} \equiv 0$ outside $U \equiv \cup_{i=1}^{n} U_{i}$

Proof. Let $\left\{\chi_{i}\right\}_{i=0}^{m}$ be a partition of unity subordinated to $\left\{U_{i}\right\}_{i=0}^{m}$ and let $u \in L^{1}\left(\Omega_{\epsilon}\right)$. We write $u=\sum_{i=0}^{m} u_{i}$, where $u_{i}=\chi_{i} u$.

For $i=0$, define

$$
\bar{u}_{0}=\left\{\begin{array}{l}
u_{0}(x), x \in \Omega_{\epsilon}, \\
0, x \in \mathbb{R}^{N} \backslash \Omega_{\epsilon} .
\end{array}\right.
$$

Since $\chi_{0} \in C_{0}^{\infty}(\Omega)$, if $u \in X\left(\Omega_{\epsilon}\right)$, then $\bar{u}_{0} \in X\left(\mathbb{R}^{N}\right)$ and $\left\|\bar{u}_{0}\right\|_{X\left(\mathbb{R}^{N}\right)} \leq c_{1}\|u\|_{X\left(\Omega_{\epsilon}\right)}$.

For $i=1, \ldots, m$, denote by $v_{i}: Q_{N}^{-} \rightarrow \mathbb{R}$ the mapping $v_{i}(y)=u\left(\Phi_{i, \epsilon}(y)\right)$. By Lemma 4.1 and Remark 4.2, we have that $v_{i} \in X\left(Q_{N}^{-}\right)$and $\left\|v_{i}\right\|_{X\left(Q_{N}^{-}\right)} \leq c_{2}\|u\|_{X\left(U_{i} \cap \Omega_{\epsilon}\right)}$, with $c_{2}$ independent of $\epsilon$.

Define $v_{i}^{*} \in X\left(Q_{N}\right)$ by

$$
v_{i}^{*}=\left\{\begin{array}{l}
v_{i}(x), x \in Q_{N}^{-} \\
v_{i}(-x), x \in Q_{N}^{+}
\end{array}\right.
$$

Thus, $\left\|v_{i}^{*}\right\|_{X\left(Q_{N}\right)} \leq 2\left\|v_{i}\right\|_{X\left(Q_{N}^{-}\right)}$. where $Q_{N}^{+}=Q_{N-1} \times(0,1)$. Then, consider $w_{i}: U_{i} \rightarrow \mathbb{R}$ given by $w_{i}(z)=v_{i}^{*}\left(\Phi_{i, \epsilon}^{-1}(z)\right)$. By Lemma 4.1 and Remark 4.2, we have that $w_{i} \in X\left(U_{i}\right)$ and $\left\|w_{i}\right\|_{X\left(U_{i}\right)} \leq c_{3}\left\|v_{i}^{*}\right\|_{X\left(Q^{N}\right)}$, with $c_{3}$ independent of $\epsilon$. Furthermore, $w_{i \mid U_{i} \cap \Omega_{\epsilon}}=u_{\mid U_{i} \cap \Omega_{\epsilon}}$ 
Finally, if we define

$$
\bar{u}_{i}=\left\{\begin{array}{l}
\chi_{i} w_{i}(x), x \in U_{i} \\
0, x \in \mathbb{R}^{N} \backslash U_{i}
\end{array}\right.
$$

we get $\bar{u}_{i} \in X\left(\mathbb{R}^{N}\right)$ and $\left\|\bar{u}_{i}\right\|_{X\left(\mathbb{R}^{N}\right)} \leq c_{4}\left\|w_{i}\right\|_{X\left(U_{i}\right)}$. Observe that $\left.\bar{u}_{i}\right|_{\Omega_{\epsilon}}=u_{i}$. Therefore, define $P_{\Omega_{\epsilon}}(u)=\sum_{i=0}^{m} \bar{u}_{i}$. Since the constants $c_{1}, c_{2}, c_{3}$ and $c_{4}$ are independent of $\epsilon$ we get the first part of the result.

To show that $P_{\Omega_{\epsilon}} u \equiv 0$ outside $U$, we just observe that $\operatorname{supp}\left(\bar{u}_{i}\right) \subset U_{i}$.

Remark 4.4. The construction of the operators $P_{\Omega_{\epsilon}}$ will permit us to construct a whole family of operators, $P_{\Omega_{\epsilon}, V}: X\left(\Omega_{\epsilon}\right) \rightarrow X(V)$, defined by $P_{\Omega_{\epsilon}, V}=R_{V} \circ P_{\Omega_{\epsilon}}$, where $R_{V}$ is the restriction operator to the open set $V$. With this notation $P_{\Omega_{\epsilon}}=P_{\Omega_{\epsilon}, \mathbb{R}^{N}}$. We also have $\left\|P_{\Omega_{\epsilon}, V}\right\|_{\mathcal{L}\left(X\left(\Omega_{\epsilon}\right), X(V)\right)} \leq C$, independent of $\epsilon$.

With the result on the extension operators we can analyze the Sobolev imbeddings.

Proposition 4.5. Let $\Omega_{\epsilon}, 0 \leq \epsilon<\epsilon_{0}$, be a family of domains satisfying conditions $(\mathbf{H})$ and (F) i). Then, the constants of the continuous imbedding $W^{1, p}\left(\Omega_{\epsilon}\right) \hookrightarrow L^{q}\left(\Omega_{\epsilon}\right)$, for $1 \leq p<N$ and $1 \leq q \leq \frac{N p}{N-p}$ or $W^{1, p}\left(\Omega_{\epsilon}\right) \hookrightarrow C^{\alpha}\left(\bar{\Omega}_{\epsilon}\right)$, for $p>N$ and $0<\alpha<1-\frac{N}{p}$, is uniformly bounded in $\epsilon$.

Proof. The result is trivially satisfied for $1 \leq q \leq p$, since the constants of the embeddings $W^{1, p}\left(\Omega_{\epsilon}\right) \hookrightarrow L^{p}\left(\Omega_{\epsilon}\right) \hookrightarrow L^{q}\left(\Omega_{\epsilon}\right)$ are all bounded uniformly in $\epsilon$.

For $1 \leq p<N$ and $p<q \leq \frac{N p}{N-p}$ we consider $u \in W^{1, p}\left(\Omega_{\epsilon}\right)$ and by Proposition 4.3. $P_{\Omega_{\epsilon}}(u) \in W^{1, p}\left(\mathbb{R}^{N}\right) \hookrightarrow L^{q}\left(\mathbb{R}^{N}\right)$ for $p \leq q \leq \frac{N p}{N-p}$. Then

$$
\|u\|_{L^{q}\left(\Omega_{\epsilon}\right)} \leq\left\|P_{\Omega_{\epsilon}}(u)\right\|_{L^{q}\left(\mathbb{R}^{N}\right)} \leq k_{1}\left\|P_{\Omega_{\epsilon}}(u)\right\|_{W^{1, p}\left(\mathbb{R}^{N}\right)} \leq k_{2}\|u\|_{W^{1, p}\left(\Omega_{\epsilon}\right)}
$$

which proves the result in this case. For the case $p>N$ we proceed in a similar way.

With similar arguments as in the proof of Proposition 4.3. we obtain a result on trace operators.

Proposition 4.6. Let $\Omega_{\epsilon}, 0 \leq \epsilon<\epsilon_{0}$, be a family of domains satisfying conditions $(\mathbf{H})$ and (F) i). Then, the constant of the trace operator $W^{1, p}\left(\Omega_{\epsilon}\right) \rightarrow L^{q}\left(\partial \Omega_{\epsilon}\right)$ for $1 \leq p<N$ and $1 \leq q \leq \frac{N p-p}{N-p}$ is bounded uniformly in $\epsilon$, that is, there exists $C$ independent of $\epsilon$, such that for all $u_{\epsilon} \in W^{1, p}\left(\Omega_{\epsilon}\right)$

$$
\left\|u_{\epsilon}\right\|_{L^{q}\left(\partial \Omega_{\epsilon}\right)} \leq C\left\|u_{\epsilon}\right\|_{W^{1, p}\left(\Omega_{\epsilon}\right)} .
$$

Moreover, if $1 \leq q<\frac{N p-p}{N-p}$, then for each $\delta>0$, there exists a $C_{\delta}$, independent of $\epsilon$, such that for all $u_{\epsilon} \in W^{1, p}\left(\Omega_{\epsilon}\right)$

$$
\left\|u_{\epsilon}\right\|_{L^{q}\left(\partial \Omega_{\epsilon}\right)} \leq C_{\delta}\left\|u_{\epsilon}\right\|_{L^{1}\left(\Omega_{\epsilon}\right)}+\delta\left\|u_{\epsilon}\right\|_{W^{1, p}\left(\Omega_{\epsilon}\right)} .
$$


Proof. Observe that $\left\|u_{\epsilon}\right\|_{L^{q}\left(\partial \Omega_{\epsilon}\right)} \leq C \sum_{i=1}^{n}\left\|u_{\epsilon}\right\|_{L^{q}\left(\partial \Omega_{\epsilon} \cap U_{i}\right)}$. Applying Lemma 4.1, we have for $1 \leq q \leq \frac{N p-p}{N-p}$

$$
\begin{gathered}
\left\|u_{\epsilon}\right\|_{L^{q}\left(\partial \Omega_{\epsilon} \cap U_{i}\right)} \leq C\left\|u_{\epsilon} \circ \Phi_{i, \epsilon}\right\|_{L^{q}\left(Q_{N-1}\right)} \leq C\left\|u_{\epsilon} \circ \Phi_{i, \epsilon}\right\|_{W^{1, p}\left(Q_{N}^{-}\right)} \\
\leq C\left\|u_{\epsilon}\right\|_{W^{1, p}\left(\Omega_{\epsilon} \cap U_{i}\right)} \leq C\left\|u_{\epsilon}\right\|_{W^{1, p}\left(\Omega_{\epsilon}\right)}
\end{gathered}
$$

from which the first inequality follows easily.

To show the second inequality, observe that if $1 \leq q<\frac{N p-p}{N-p}$ by interpolation we have

$$
\begin{gathered}
\left\|u_{\epsilon} \circ \Phi_{i, \epsilon}\right\|_{L^{q}\left(Q_{N-1}\right)} \leq C_{\delta}\left\|u \circ \Phi_{i, \epsilon}\right\|_{L^{1}\left(Q_{N}^{-}\right)}+\delta\left\|u \circ \Phi_{i, \epsilon}\right\|_{W^{1, p}\left(Q_{N}^{-}\right)} \\
\leq C C_{\delta}\left\|u_{\epsilon}\right\|_{L^{1}\left(\Omega_{\epsilon} \cap U_{i}\right)}+C \cdot \delta\left\|u_{\epsilon}\right\|_{W^{1, p}\left(\Omega_{\epsilon} \cap U_{i}\right)}
\end{gathered}
$$

from where the last inequality follows easily.

Finally, we can prove

Corollary 4.7. In the same conditions of Proposition 4.6, if $v_{\epsilon} \rightarrow v$ weakly in $W^{1, p}(U)$, then, for all $1 \leq q<\frac{N p-p}{N-p}$ we have $\left\|v_{\epsilon}-v\right\|_{L^{q}\left(\partial \Omega_{\epsilon}\right)} \rightarrow 0$.

Proof. Observe that if $v_{\epsilon} \rightarrow v \mathrm{w}-W^{1, p}(U)$, then $v_{\epsilon} \rightarrow v$ in $L^{1}(U)$ and $\left\|v_{\epsilon}\right\|_{W^{1, p}(U)},\|v\|_{W^{1, p}(U)} \leq$ $C$. Hence, using (4.3), we have, for $\delta>0$, arbitrarily small

$$
\left\|v_{\epsilon}-v\right\|_{L^{q}\left(\partial \Omega_{\epsilon}\right)} \leq C_{\delta}\left\|v_{\epsilon}-v\right\|_{L^{1}\left(\Omega_{\epsilon}\right)}+\delta\left\|v_{\epsilon}-v\right\|_{W^{1, p}\left(\Omega_{\epsilon}\right)} .
$$

Hence,

$$
\limsup _{\epsilon \rightarrow 0}\left\|v_{\epsilon}-v\right\|_{L^{q}\left(\partial \Omega_{\epsilon}\right)} \leq C \cdot \delta .
$$

Since $\delta>0$ is arbitrarily small, we obtain the results.

Once these results on extension operators, Sobolev embeddings and trace operators have been established, we will prove other technical results also needed in the proof of the main result.

Lemma 4.8. Assume $\mathbf{( H )}$ is satisfied and let $1<p<\infty$. There exists a function $c(\epsilon)$ with $c(\epsilon) \rightarrow 0$ as $\epsilon \rightarrow 0$, such that for any $u \in W^{1, p}(U)$ and for all $1 \leq q \leq p, i=1, \ldots, n$, we have

$$
\left\|u \circ \phi_{i, \epsilon}-u \circ \phi_{i, 0}\right\|_{L^{q}\left(Q_{N-1}\right)} \leq c(\epsilon)\|u\|_{W^{1, p}(U)} .
$$

Moreover, if hypothesis $(\mathbf{F})$ i) is also satisfied, then (4.4) also holds for $1 \leq q<(\mathrm{Np}-$ $p) /(N-p)$.

Proof. To prove statement (4.4) for $1 \leq q \leq p$ it will be enough to show it for $q=p$, since using Hölder inequality we will prove it for all $1 \leq q \leq p$. Hence,

$$
\int_{Q_{N-1}}\left|u \circ \phi_{i, \epsilon}\left(x^{\prime}\right)-u \circ \phi_{i, 0}\left(x^{\prime}\right)\right|^{p} d x^{\prime}=\int_{Q_{N-1}}\left|u \circ \Phi_{i}\left(x^{\prime}, \rho_{i, \epsilon}\left(x^{\prime}\right)\right)-u \circ \Phi_{i}\left(x^{\prime}, 0\right)\right|^{p} d x^{\prime}
$$




$$
\begin{gathered}
=\int_{Q_{N-1}}\left|\int_{0}^{\rho_{i, \epsilon}\left(x^{\prime}\right)} \frac{\partial\left(u \circ \Phi_{i}\right)}{\partial x_{N}}\left(x^{\prime}, x_{N}\right) d x_{N}\right|^{p} d x^{\prime} \\
\leq \int_{Q_{N-1}}\left|\rho_{i, \epsilon}\left(x^{\prime}\right)\right|^{p / p^{\prime}} \int_{0}^{\rho_{i, \epsilon}\left(x^{\prime}\right)}\left|\frac{\partial\left(u \circ \Phi_{i}\right)}{\partial x_{N}}\left(x^{\prime}, x_{N}\right)\right|^{p} d x_{N} d x^{\prime} \\
\leq\left\|\rho_{i, \epsilon}\right\|_{L^{\infty}\left(Q_{N-1}\right)}^{p / p^{\prime}} \int_{Q_{N-1}} \int_{0}^{\rho_{i, \epsilon}\left(x^{\prime}\right)}\left|\frac{\partial\left(u \circ \Phi_{i}\right)}{\partial x_{N}}\left(x^{\prime}, x_{N}\right)\right|^{p} d x_{N} d x^{\prime} .
\end{gathered}
$$

Noticing that $\Phi_{i}$ is a smooth Lipschitz diffeomorphism and using the chain rule in the last expression, we easily get that

$$
\int_{Q_{N-1}} \int_{0}^{\rho_{i, \epsilon}\left(x^{\prime}\right)}\left|\frac{\partial\left(u \circ \Phi_{i}\right)}{\partial x_{N}}\left(x^{\prime}, x_{N}\right)\right|^{p} d x_{N} d x^{\prime} \leq C\|u\|_{W^{1, p}(U)}^{p}
$$

which shows the first part of the result with $c(\epsilon)=C\left\|\rho_{i, \epsilon}\right\|_{L^{\infty}\left(Q_{N-1}\right)}^{p / p^{\prime}}$.

Moreover, assuming (F) i) holds, from Proposition 4.6] we get that both $\left\|u \circ \phi_{i, \epsilon}\right\|_{L^{r}\left(Q_{N-1}\right)}, \| u \circ$ $\phi_{i, 0}\left\|_{L^{r}\left(Q_{N-1}\right)} \leq C\right\| u \|_{W^{1, p}(U)}$. Hence, using this uniform bound, inequality (4.4) for $q=1$ and interpolating between $L^{1}\left(Q_{N-1}\right)$ and $L^{(N p-p) /(N-p)}\left(Q_{N-1}\right)$, we prove the last part of the Lemma.

In the following result, we obtain the $E$-convergence of a suitable sequence of functions.

Lemma 4.9. Assume $\mathbf{( H )}$ is satisfied. Let $u_{\epsilon} \in H^{1}\left(\Omega_{\epsilon}\right)$ such that $\left\|u_{\epsilon}\right\|_{H^{1}\left(\Omega_{\epsilon}\right)} \leq M$. Then,

i) There exists a subsequence, denoted by $u_{\epsilon_{k}}$ and $u_{0} \in H^{1}(\Omega)$ such that $u_{\epsilon_{k}} \stackrel{E}{\longrightarrow} u_{0}$.

ii) If we also assume that $(\mathbf{F})$ i) holds, then, there exists a subsequence $\epsilon_{k}$, and a function $u \in H^{1}(U)$ such that $P_{\Omega_{\epsilon_{k}}, U}\left(u_{\epsilon_{k}}\right) \rightarrow u, w-H^{1}(U)$ and $u_{\epsilon_{k}} \stackrel{E}{\longrightarrow} u_{\left.\right|_{\Omega}}$.

Proof. i) With an standard argument we can get a subsequence, that we still denote by $u_{\epsilon}$ and a function $u_{0} \in H^{1}(\Omega)$, with the property that $u_{\left.\epsilon\right|_{K}} \rightarrow u_{\left.0\right|_{K}}$ for all $K \subset \subset \Omega$. To show that, for this subsequence, $u_{\epsilon} \stackrel{E}{\longrightarrow} u_{0}$, we observe that by Proposition 3.3 it is enough to show that $\left(u_{\epsilon}, E_{\epsilon} v\right)_{H^{1}\left(\Omega_{\epsilon}\right)} \rightarrow\left(u_{0}, v\right)_{H^{1}(\Omega)}$ for any $v \in H^{1}(\Omega)$. In fact, for any $K \subset \subset \Omega$, we have

$$
\left(u_{\epsilon}, E_{\epsilon} v\right)_{H^{1}\left(\Omega_{\epsilon}\right)}-\left(u_{0}, v\right)_{H^{1}(\Omega)}=\left(u_{\epsilon}-u_{0}, v\right)_{H^{1}(K)}+\left(u_{\epsilon}, E_{\epsilon} v\right)_{H^{1}\left(\Omega_{\epsilon} \backslash K\right)}-\left(u_{0}, v\right)_{H^{1}(\Omega \backslash K)} .
$$

Since we have

a) $\left(u_{\epsilon}-u_{0}, v\right)_{H^{1}(K)} \rightarrow 0$

b) $\left|\left(u_{0}, v\right)_{H^{1}(\Omega \backslash K)}\right| \leq\left\|u_{0}\right\|_{H^{1}(\Omega \backslash K)}\|v\|_{H^{1}(\Omega \backslash K)} \leq M\|v\|_{H^{1}(\Omega \backslash K)}$, and

c) $\left|\left(u_{\epsilon}, E_{\epsilon} v\right)_{H^{1}\left(\Omega_{\epsilon} \backslash K\right)}\right| \leq\left\|u_{\epsilon}\right\|_{H^{1}\left(\Omega_{\epsilon} \backslash K\right)}\left\|E_{\epsilon} v\right\|_{H^{1}\left(\Omega_{\epsilon} \backslash K\right)} \leq M\left\|E_{\epsilon} v\right\|_{H^{1}\left(\Omega_{\epsilon} \backslash K\right)} \rightarrow M\|v\|_{H^{1}(\Omega \backslash K)}$, as $\epsilon \rightarrow 0$,

we obtain

$$
\limsup _{\epsilon \rightarrow 0}\left|\left(u_{\epsilon}, E_{\epsilon} v\right)_{H^{1}\left(\Omega_{\epsilon}\right)}-\left(u_{0}, v\right)_{H^{1}(\Omega)}\right| \leq 2 M\|v\|_{H^{1}(\Omega \backslash K)}
$$


Since $K \subset \subset \Omega$ is arbitrary and therefore the measure of $|\Omega \backslash K|$ is arbitrarily small and the function $v$ is fixed, we get that the limit above necessarily must be zero and we get the result.

ii) Since $\left\|u_{\epsilon}\right\|_{H^{1}\left(\Omega_{\epsilon}\right)} \leq M$ and the family of maps $P_{\Omega_{\epsilon}, U}$ are uniformly bounded in $\epsilon$, we have that $\left\|P_{\Omega_{\epsilon}, U}\left(u_{\epsilon}\right)\right\|_{H^{1}(U)}$ is uniformly bounded. Hence, we can get a function $u \in H^{1}(U)$ and a subsequence, that we denote again by $P_{\Omega_{\epsilon}, U}\left(u_{\epsilon}\right)$ such that $P_{\Omega_{\epsilon}, U}\left(u_{\epsilon}\right) \rightarrow u$ weakly in $H^{1}(U)$. To prove that we also have $u_{\epsilon} \stackrel{E}{\longrightarrow} u_{\left.\right|_{\Omega}}$ we follow the same argument as in i)

\section{Proof of the main results}

In this section we will provide a proof of Theorem 2.4 and Theorem 2.6. For this, we will need to prove a series of results and at the end of the section we indicate how this results actually prove the main theorems.

We keep the notation of the previous sections and in particular we consider a family of domains $\Omega_{\epsilon}$ satisfying conditions $(\mathbf{H})$ and $(\mathbf{F})$. We will obtain the convergence of the equilibria of the problem in $\Omega_{\epsilon}$ to the solutions of the problem in $\Omega$.

In terms of the nonlinearities, taking into account Remark 2.5, we will assume that $f$ and $g$ satisfy conditions (2.4) and (2.5) , respectively.

Consider the family of spaces $H^{1}\left(\Omega_{\epsilon}\right)$ and $H^{1}(\Omega)$ with their usual norms. We define the family of linear operators $E_{\epsilon}: H^{1}(\Omega) \rightarrow H^{1}\left(\Omega_{\epsilon}\right)$ given by $E_{\epsilon}=R_{\epsilon} \circ P$, where $P$ is a linear and continuous operator $P: H^{1}(\Omega) \rightarrow H^{1}\left(\mathbb{R}^{N}\right)$ that extends a function $u$ defined in $\Omega$ to a function defined in $\mathbb{R}^{N}$ and $R_{\epsilon}$ is the restriction to $\Omega_{\epsilon}, R_{\epsilon}(w)=w_{\mid \Omega_{\epsilon}}$. Considering this definition and the fact that from $(\mathbf{H}),\left|\Omega_{\epsilon} \backslash \Omega\right| \rightarrow 0$ and $\left|\Omega \backslash \Omega_{\epsilon}\right| \rightarrow 0$ when $\epsilon \rightarrow 0$, we get that

$$
\left\|E_{\epsilon}(u)\right\|_{H^{1}\left(\Omega_{\epsilon}\right)} \rightarrow\|u\|_{H^{1}(\Omega)} .
$$

We also have, $\left\|E_{\epsilon}\right\| \leq\left\|R_{\epsilon}\right\| \cdot\|P\| \leq\|P\|$, independent of $\epsilon$.

With respect to the function $\gamma$ defined in Section 2 we have the following

Lemma 5.1. Assume hypotheses $(\mathbf{H})$ and $(\mathbf{F})$ hold. Then, for any function $f \in W^{1,1}(U)$ and for any $x_{0} \in \partial \Omega, r>0$ small, we get

$$
\int_{\partial \Omega_{\epsilon} \cap B\left(x_{0}, r\right)} f \rightarrow \int_{\partial \Omega \cap B\left(x_{0}, r\right)} f \gamma
$$

In particular, we also have

$$
\int_{\partial \Omega_{\epsilon}} f \rightarrow \int_{\partial \Omega} f \gamma
$$

Proof. Consider the finite cover $\left\{U_{i}\right\}_{i=0}^{m}$, such that $\Omega_{\epsilon} \subset \cup_{i=0}^{m} U_{i}$. Without loss of generality we may assume that $B\left(x_{0}, r\right) \subset U_{i}$ for some $i \in\{1, \ldots, n\}$. If we denote by 


$$
\begin{aligned}
& B_{i}=\Phi_{i}^{-1}\left(B\left(x_{0}, r\right) \cap \partial \Omega\right) \text {, then } \\
& \qquad \int_{B\left(x_{0}, r\right) \cap \partial \Omega_{\epsilon}} f-\int_{B\left(x_{0}, r\right) \cap \partial \Omega} f \gamma=\int_{Q_{N-1} \cap B_{i}} f \circ \phi_{i, \epsilon} . J \phi_{i, \epsilon}-(f \gamma) \circ \phi_{i, 0} . J \phi_{i, 0} \\
& \quad=\int_{Q_{N-1} \cap B_{i}}\left(f \circ \phi_{i, \epsilon}-f \circ \phi_{i}\right) . J \phi_{i, \epsilon}+\int_{Q_{N-1} \cap B_{i}} f \circ \phi_{i, 0} \cdot\left(J \phi_{i, \epsilon}-\gamma_{i}\right)
\end{aligned}
$$

Using the definition of $\gamma_{i}$ given in hypothesis (F) ii) and the fact that $\rho_{i, \epsilon} \rightarrow 0$ uniformly in $Q_{N-1}$, we obtain that the integrals goes to zero.

As a consequence of this result, we get

Corollary 5.2. The function $\gamma$ is independent of the parameterization chosen and therefore it is unique. Moreover, $\gamma \geq 1$.

Proof. Suppose that $\gamma$ depends on the parametrization. Then there will exist $\gamma$ and $\tilde{\gamma}$, both satisfying Lemma 5.1. Hence

$$
\int_{\partial \Omega} f \gamma=\int_{\partial \Omega} f \tilde{\gamma}, \text { for all } f \in C_{0}^{\infty}\left(\mathbb{R}^{N}\right)
$$

This implies that $\gamma=\tilde{\gamma}$ almost everywhere in $\partial \Omega$.

Moreover, taking $f \equiv 1$ in Lemma [5.1, we get that $\left|\partial \Omega_{\epsilon} \cap B\left(x_{0}, r\right)\right| \rightarrow\|\gamma\|_{L^{1}\left(\partial \Omega \cap B\left(x_{0}, r\right)\right)}$. But, since $\partial \Omega_{\epsilon}$ approaches $\partial \Omega$, then by the upper semicontinuity of the measure, we have that $\liminf _{\epsilon \rightarrow 0}\left|\partial \Omega_{\epsilon} \cap B\left(x_{0}, r\right)\right| \geq\left|\partial \Omega \cap B\left(x_{0}, r\right)\right|$. This implies that $\|\gamma\|_{L^{1}\left(\partial \Omega \cap B\left(x_{0}, r\right)\right)} \geq$ $\left|\partial \Omega \cap B\left(x_{0}, r\right)\right|$, for all $x_{0} \in \partial \Omega$ and for all $r>0$ small. This implies that $\gamma \geq 1$.

Lemma 5.3. Assume $(\mathbf{H})$ and $(\mathbf{F}) i)$ are satisfied. Let $u_{\epsilon}$ be a bounded sequence in $H^{1}\left(\Omega_{\epsilon}\right)$ and let $w_{\epsilon}$ be given by $w_{\epsilon}=A_{\epsilon}^{-1} h_{\epsilon}\left(u_{\epsilon}\right)$. Then $w_{\epsilon}$ is also a bounded sequence in $H^{1}\left(\Omega_{\epsilon}\right)$.

Proof. Recall that saying that $w_{\epsilon}=A_{\epsilon}^{-1} h_{\epsilon}\left(u_{\epsilon}\right)$ is equivalent to saying that $w_{\epsilon}$ is the weak solution of $-\Delta w_{\epsilon}+w_{\epsilon}=f\left(x, u_{\epsilon}\right)$ in $\Omega_{\epsilon}$ with boundary condition $\frac{\partial w_{\epsilon}}{\partial n}+g\left(x, u_{\epsilon}\right)=0$ on $\partial \Omega_{\epsilon}$. Hence, we get

$$
\left\|w_{\epsilon}\right\|_{H^{1}\left(\Omega_{\epsilon}\right)}^{2}=\int_{\Omega_{\epsilon}} f\left(x, u_{\epsilon}\right) w_{\epsilon}-\int_{\partial \Omega_{\epsilon}} g\left(x, u_{\epsilon}\right) w_{\epsilon} .
$$

Using the boundedness of $f$ and $g$ given by (2.4) and (2.5), and applying the embeddings and trace theorems obtained in Proposition 4.6 and 4.5, we easily get the result.

Now, we prove the convergence of nonlinear part.

Proposition 5.4. Assume $(\mathbf{H})$ and $(\mathbf{F})$ are satisfied. Let $w_{\epsilon}, u_{\epsilon} \in H^{1}\left(\Omega_{\epsilon}\right)$, such that $P_{\Omega_{\epsilon}, U}\left(u_{\epsilon}\right) \rightarrow u, w-H^{1}(U)$ and $P_{\Omega_{\epsilon}, U}\left(w_{\epsilon}\right) \rightarrow w, w-H^{1}(U)$. Then,

$$
\begin{aligned}
\int_{\Omega_{\epsilon}} f\left(x, u_{\epsilon}\right) w_{\epsilon} & \rightarrow \int_{\Omega} f(x, u) w \\
\int_{\partial \Omega_{\epsilon}} g\left(x, u_{\epsilon}\right) w_{\epsilon} & \rightarrow \int_{\partial \Omega} g(x, u) w \gamma
\end{aligned}
$$


This implies that $\left\langle h_{\epsilon}\left(u_{\epsilon}\right), w_{\epsilon}\right\rangle \rightarrow\langle h(u), w\rangle$.

Proof. From (2.4) and using the convergence of $u_{\epsilon}, w_{\epsilon}$ we easily get the first statement of (5.1).

For the second statement, observe that

$$
\begin{gathered}
\left|\int_{\partial \Omega_{\epsilon}} g\left(x, u_{\epsilon}\right) w_{\epsilon}-\int_{\partial \Omega} g(x, u) w \gamma\right| \leq \int_{\partial \Omega_{\epsilon}}\left|g\left(x, u_{\epsilon}\right)-g(x, u)\right|\left|w_{\epsilon}\right| \\
+\int_{\partial \Omega_{\epsilon}}|g(x, u)|\left|w_{\epsilon}-w\right|+\left|\int_{\partial \Omega_{\epsilon}} g(x, u) w-\int_{\partial \Omega} g(x, u) w \gamma\right| \\
\leq C\left\|u_{\epsilon}-u\right\|_{L^{2}\left(\partial \Omega_{\epsilon}\right)}\left\|w_{\epsilon}\right\|_{L^{2}\left(\partial \Omega_{\epsilon}\right)}+C\left\|w_{\epsilon}-w\right\|_{L^{1}\left(\partial \Omega_{\epsilon}\right)}+\left|\int_{\partial \Omega_{\epsilon}} g(x, u) w-\int_{\partial \Omega} g(x, u) w \gamma\right| \rightarrow 0
\end{gathered}
$$

where we use Corollary 4.7 to prove that the first two terms go to 0 and Lemma 5.1 for the last one.

Now, we are in conditions to prove

Proposition 5.5. Assume $(\mathbf{H})$ and $(\mathbf{F})$ are satisfied. Then, $A_{\epsilon}^{-1} h_{\epsilon} \stackrel{C C}{\longrightarrow} A^{-1} h$.

Proof. In order to prove the compact converge of $A_{\epsilon}^{-1} h_{\epsilon}$ to $A^{-1} h$, we need to verify that i) $A_{\epsilon}^{-1} h_{\epsilon}$ is a compact operator for each $\epsilon>0$ fixed,

ii) $\left\{A_{\epsilon}^{-1} h\left(u_{\epsilon}\right)\right\}_{0 \leq \epsilon \leq \epsilon_{0}}$ is an E-precompact family whenever $\left\|u_{\epsilon}\right\|_{H^{1}\left(\Omega_{\epsilon}\right)}$ is bounded.

iii) $A_{\epsilon}^{-1} h_{\epsilon}\left(u_{\epsilon}\right) \stackrel{E}{\longrightarrow} A^{-1} h\left(u_{0}\right)$, if $u_{\epsilon} \stackrel{E}{\longrightarrow} u_{0}$.

Let us show each of the three points above.

i) For each $\epsilon>0$ fixed, since $h: H^{1}\left(\Omega_{\epsilon}\right) \rightarrow H^{-\alpha}\left(\Omega_{\epsilon}\right)$ for some $1 / 2<\alpha<1$ and $A_{\epsilon}^{-1}$ : $H^{-\alpha}\left(\Omega_{\epsilon}\right) \rightarrow H^{2-\alpha}\left(\Omega_{\epsilon}\right)$ are continuous maps and using the compact imbedding of $H^{2-\alpha}\left(\Omega_{\epsilon}\right)$ in $H^{1}\left(\Omega_{\epsilon}\right)$, with $2-\alpha>1$, we get that the $A_{\epsilon}^{-1} h_{\epsilon}$ is compact.

ii) Let $u_{\epsilon} \in H^{1}\left(\Omega_{\epsilon}\right)$ be such that $\left\|u_{\epsilon}\right\|_{H^{1}\left(\Omega_{\epsilon}\right)} \leq C$. In particular, from Lemma 4.9] ii) we get a subsequence and a function $u \in H^{1}(U)$ such that $P_{\Omega_{\epsilon_{k}}, U}\left(u_{\epsilon_{k}}\right) \rightarrow u, \mathrm{w}-H^{1}(U)$ and $u_{\epsilon_{k}} \stackrel{E}{\longrightarrow} u_{0}$ where $u_{0}=u_{\left.\right|_{\Omega}}$.

Consider now $w_{\epsilon_{k}}=A_{\epsilon_{k}}^{-1} h_{\epsilon_{k}}\left(u_{\epsilon_{k}}\right)$. Then, by Lemma 5.3, $\left\|w_{\epsilon_{k}}\right\|_{H^{1}\left(\Omega_{\epsilon_{k}}\right)}$ is a bounded sequence and therefore, again by Lemma $4.9 \mathrm{ii}$ ) we get a subsequence, that we denote again by $w_{\epsilon_{k}}$ and a function $w \in H^{1}(U)$ such that $P_{\Omega_{\epsilon_{k}}, U}\left(w_{\epsilon_{k}}\right) \rightarrow w, \mathrm{w}-H^{1}(U)$ and $w_{\epsilon_{k}} \stackrel{E}{\longrightarrow} w_{0}$ where $w_{0}=w_{\left.\right|_{\Omega}}$.

Let us show now that, as a matter of fact, we have $w_{0}=A^{-1} h\left(u_{0}\right)$. For this, notice that since $w_{\epsilon_{k}} \stackrel{E}{\longrightarrow} w_{0}$, we have that for any $v \in H^{1}(\Omega),\left(w_{\epsilon_{k}}, E_{\epsilon_{k}} v\right)_{H^{1}\left(\Omega_{\epsilon_{k}}\right)} \rightarrow\left(w_{0}, v\right)_{H^{1}(\Omega)}$. But, $\left(w_{\epsilon_{k}}, E_{\epsilon_{k}} v\right)_{H^{1}\left(\Omega_{\epsilon_{k}}\right)}=\left\langle h_{\epsilon_{k}}\left(u_{\epsilon_{k}}\right), E_{\epsilon_{k}} v\right\rangle \rightarrow\left\langle h\left(u_{0}\right), v\right\rangle=\left(A^{-1} h\left(u_{0}\right), v\right)_{H^{1}(\Omega)}$, where we have used Proposition [5.4, Hence, $w_{0}=A^{-1} h\left(u_{0}\right)$.

Now, we prove $\left\|w_{\epsilon_{k}}\right\|_{H^{1}\left(\Omega_{\epsilon_{k}}\right)} \rightarrow\left\|w_{0}\right\|_{H^{1}(\Omega)}$. For this, observe that $\left\|w_{\epsilon_{k}}\right\|_{H^{1}\left(\Omega_{\epsilon_{k}}\right)}^{2}=\left\langle h_{\epsilon_{k}}\left(u_{\epsilon_{k}}\right), w_{\epsilon_{k}}\right\rangle \rightarrow$ $\left\langle h\left(u_{0}\right), w\right\rangle=\left\|w_{0}\right\|_{H^{1}(\Omega)}^{2}$, where we have used again Proposition [5.4. The convergence of 
the norms and the weak E-convergence of the sequence, imply, using Proposition 3.4 that $w_{\epsilon_{k}} \stackrel{E}{\longrightarrow} w_{0}$.

iii) If we assume now that $u_{\epsilon} \stackrel{E}{\longrightarrow} u_{0}$, then we have that $\left\|u_{\epsilon}\right\|_{H^{1}\left(\Omega_{\epsilon}\right)} \leq M$. In particular, for any sequence $\epsilon_{k} \rightarrow 0$, we can extract another subsequence, that we denote it also as $\epsilon_{k}$, such that following the argument made above to prove ii), we have, that $P_{\Omega_{\epsilon_{k}}, U}\left(u_{\epsilon_{k}}\right) \rightarrow u, u_{0}=u_{\left.\right|_{\Omega}}$ and, for this subsequence, $A_{\epsilon_{k}}^{-1} h\left(u_{\epsilon_{k}}\right) \stackrel{E}{\longrightarrow} A^{-1} h\left(u_{0}\right)$. Since this has been proved for any sequence, then we obtain the $E$-convergence for the the whole family, that is $A_{\epsilon}^{-1} h\left(u_{\epsilon}\right) \stackrel{E}{\longrightarrow} A^{-1} h\left(u_{0}\right)$.

The compact convergence of $A_{\epsilon}^{-1} h_{\epsilon}$ to $A^{-1} h$ given by the previous proposition will guarantee us with the upper semicontinuity of the set of equilibria. More precisely,

Corollary 5.6. If $(\mathbf{H})$ and $(\mathbf{F})$ are satisfied, then for any family of equilibria $\left\{u_{\epsilon}^{*}\right\}_{0<\epsilon \leq \epsilon_{0}}$ of (1.1) there exists an equilibrium point $u_{0}^{*}$ of (1.2) and a subsequence of $\left\{u_{\epsilon}^{*}\right\}$ that E-converges to $u_{0}^{*}$.

Proof. Apply Theorem 3.8 .

We can also prove

Proposition 5.7. If $(\mathbf{H})$ and $(\mathbf{F})$ are satisfied, then there exists $0<\alpha<1$ and $M>0$ such that $\left\|u_{\epsilon}^{*}\right\|_{C^{\alpha}\left(\bar{\Omega}_{\epsilon}\right)},\left\|u_{0}^{*}\right\|_{C^{\alpha}(\bar{\Omega})} \leq M$, for any $u_{\epsilon}^{*}$, solution of (1.1), $0 \leq \epsilon \leq \epsilon_{0}$ and any $u_{0}^{*}$, solution of (1.2).

In particular, if $u_{\epsilon}^{*} \stackrel{E}{\longrightarrow} u_{0}^{*}$, then we also have

$$
\left\|u_{\epsilon}^{*}-E_{\epsilon} u_{0}^{*}\right\|_{C^{\beta}\left(\bar{\Omega}_{\epsilon}\right)} \rightarrow 0
$$

for any $0<\beta<\alpha$.

Proof. That the solutions of (1.2) are Hölder continuous, follows from standard elliptic regularity theory. Notice that if $u_{0}^{*}$ is a solution of (1.2) and we define $f_{u_{0}^{*}}(x)=f\left(x, u_{0}^{*}(x)\right)$ and $g_{u_{0}^{*}}(x)=g\left(x, u_{0}^{*}(x)\right)$, then, $u_{0}^{*}$ is the unique solution of the problem

$$
\begin{cases}-\Delta u+u=f_{u_{0}^{*}}(x), & \text { in } \Omega \\ \frac{\partial u}{\partial n}=-g_{u_{0}^{*}}(x), & \text { on } \partial \Omega .\end{cases}
$$

But, since $f$ and $g$ are bounded functions, then $f_{u_{0}^{*}} \in L^{\infty}(\Omega), g_{u_{0}^{*}} \in L^{\infty}(\partial \Omega)$ and elliptic regularity theory implies that $u_{0}^{*}$ is Hölder continuous and its norm in the Hölder space will be determined by $\Omega$ and $\left\|f_{u_{0}^{*}}\right\|_{L^{\infty}(\Omega)}$ and $\left\|g_{u_{0}^{*}}\right\|_{L^{\infty}(\partial \Omega) \text {. }}$.

To obtain the uniform bound of the solutions of (1.1), we start by showing that $\left\|u_{\epsilon}^{*}\right\|_{L^{\infty}\left(\Omega_{\epsilon}\right)}$ is uniformly bounded in $\epsilon$. Since by (2.4) and (2.5), $|f(x, u)|,|g(x, u)| \leq C$, then by comparison principles, we have that $\left|u_{\epsilon}^{*}(x)\right| \leq C\left|U_{\epsilon}(x)\right|$ where $U$ is the solution of

$$
\begin{cases}-\Delta U_{\epsilon}+U_{\epsilon}=1, & \text { in } \Omega_{\epsilon} \\ \frac{\partial U_{\epsilon}}{\partial n}=1, & \text { on } \partial \Omega_{\epsilon} .\end{cases}
$$


If we multiply (5.3) by $\left(U_{\epsilon}-k\right)^{+}$, where $k>0$, and integrate by parts, we obtain

$$
\int_{\Omega_{\epsilon}}\left|\nabla\left(U_{\epsilon}-k\right)^{+}\right|^{2}+\int_{\Omega_{\epsilon}}\left|\left(U_{\epsilon}-k\right)^{+}\right|^{2}+(k-1) \int_{\Omega_{\epsilon}}\left(U_{\epsilon}-k\right)^{+}=\int_{\partial \Omega_{\epsilon}}\left(U_{\epsilon}-k\right)^{+}
$$

Moreover, by the trace theorem obtained in Proposition 4.6 and if we define $A_{k, \epsilon}=\left\{x \in \Omega_{\epsilon}\right.$ : $\left.U_{\epsilon}(x)>k\right\}$, we get $\left\|\left(U_{\epsilon}-k\right)^{+}\right\|_{L^{1}\left(\partial \Omega_{\epsilon}\right)} \leq C\left\|\left(U_{\epsilon}-k\right)^{+}\right\|_{W^{1,1}\left(\Omega_{\epsilon}\right)}=C\left\|\left(U_{\epsilon}-k\right)^{+}\right\|_{W^{1,1}\left(A_{k, \epsilon}\right)} \leq$ $C\left|A_{k, \epsilon}\right|^{\frac{1}{2}}\left\|\left(U_{\epsilon}-k\right)^{+}\right\|_{H^{1}\left(\Omega_{\epsilon}\right)}$. Hence, if $k>1$, we get $\left\|\left(U_{\epsilon}-k\right)^{+}\right\|_{H^{1}\left(\Omega_{\epsilon}\right)} \leq C\left|A_{k, \epsilon}\right|^{\frac{1}{2}}$. Moreover, by the Sobolev embeddings of Proposition 4.5, we get that there exists a constant $C$ independent of $\epsilon, k$ and $U_{\epsilon}$, such that

$$
\begin{aligned}
\left\|\left(U_{\epsilon}-k\right)^{+}\right\|_{L^{1}\left(A_{k, \epsilon}\right)} & \leq\left|A_{k, \epsilon}\right|^{\frac{N+2}{2 N}}\left\|\left(U_{\epsilon}-k\right)^{+}\right\|_{L^{\frac{2 N}{N-2}}\left(A_{k, \epsilon}\right)}=\left|A_{k, \epsilon}\right|^{\frac{N+2}{2 N}}\left\|\left(U_{\epsilon}-k\right)^{+}\right\|_{L^{\frac{2 N}{N-2}}\left(\Omega_{\epsilon}\right)} \\
& \leq C\left|A_{k, \epsilon}\right|^{\frac{N+2}{2 N}}\left\|\left(U_{\epsilon}-k\right)^{+}\right\|_{H^{1}\left(\Omega_{\epsilon}\right)} \leq C\left|A_{k, \epsilon}\right|^{1+\frac{1}{N}}
\end{aligned}
$$

This last estimate allows us to apply Lemma 5.1 from [14 and obtain the $L^{\infty}$ uniform bound for $U_{\epsilon}$, which implies the uniform $L^{\infty}$ bound for all $u_{\epsilon}^{*}$.

Observe that the key ingredient in the proof above is the uniform Sobolev embeddings and trace theorem obtained in Proposition 4.5 and Proposition 4.6, respectively. To obtain the uniform Hölder estimates on $u_{\epsilon}^{*}$ we follow the same ideas. As a matter of fact, for a fixed $\epsilon$ we can apply Lemma B.1 v) of [7] and obtain that $u_{\epsilon}^{*} \in C^{\alpha}\left(\bar{\Omega}_{\epsilon}\right)$ for certain $0<\alpha<1$. To conclude that the Hölder norm is uniformly bounded in $\epsilon$, we realize that the estimate (B.11) of [7] depends on the domain through the measure of the domain $\left|\Omega_{\epsilon}\right|$, which is controlled in our case, and through the constants $C$ that appear in (B.10). This constants come exactly from the Sobolev embeddings and trace theorems which are also controlled in our case. Hence, we can obtain estimate (B.11) where all the constants are independent of $\epsilon$. Applying Theorem 7.2 from [14] we obtain the uniform Hölder estimates on the solutions $u_{\epsilon}^{*}$.

The last part of the proposition is obtained through the compact embedding of $C^{\alpha}\left(\bar{\Omega}_{\epsilon}\right) \hookrightarrow$ $C^{\beta}\left(\bar{\Omega}_{\epsilon}\right)$ for all $\beta<\alpha$.

We also have a kind of converse result of Corollary [5.6, in the case where the equilibrium of the limiting problem is hyperbolic.

Corollary 5.8. If $(\mathbf{H})$ and $(\mathbf{F})$ are satisfied and if $u_{0}^{*}$ is a hyperbolic equilibrium point of (1.2) then there exists $\left\{u_{\epsilon}^{*}\right\}$ equilibrium points of (1.1) such that $\left\{u_{\epsilon}^{*}\right\} E$-converges to $u_{0}^{*}$.

Proof. Since $u_{0}^{*}$ is a hyperbolic equilibrium point of (1.2),$u_{0}^{*}$ isolated. Then there exists $\delta>0$ such that $u_{0}^{*}$ is the unique equilibrium point in $B\left(u_{0}^{*}, \delta\right)$ and its index, relatively to the map $A_{0}^{-1} h$, satisfies $\left|\operatorname{ind}\left(u_{0}^{*}, A_{0}^{-1} h\right)\right|=1$. We refer to [13] for a definition of the index.

Since $A_{\epsilon}^{-1} h_{\epsilon}$ compactly converges to $A_{0}^{-1} h$ then, by Theorem 3 in [19], we get that $\left|\operatorname{ind}\left(B\left(E_{\epsilon} u_{0}^{*}, \delta\right), A_{\epsilon}^{-1} h_{\epsilon}\right)\right|=1$. In consequence, for each $\epsilon$ there exists at least one equilibrium point $u_{\epsilon}^{*}$ of (1.1) in $B\left(E_{\epsilon} u_{0}^{*}, \delta\right)$. If $u_{\epsilon}^{*}$ does not $E$-converges to $u_{0}^{*}$, then there will exist a subsequence $u_{\epsilon_{k}}^{*}$ such that no other sub-subsequence can $E$-converge to $u_{0}^{*}$. But from 
Corollary [5.6, we will have that there exists $\tilde{u}_{0}$, an equilibrium point of the limiting equation (1.2) and a subsequence of $u_{\epsilon_{k}}^{*}$ which $E$-converges to $\tilde{u}_{0}$. We will necessarily have that $\tilde{u}_{0} \in B\left(u_{0}^{*}, \delta\right)$. But this will imply that $\tilde{u}_{0}=u_{0}^{*}$, which is a contradiction.

Remark 5.9. In case all equilibrium points of the limiting equation (1.2) are hyperbolic, we have that the equilibrium points are all isolated, there are a finite number of them and from Corollary 5.6 and 5.8 we get that the family $\mathcal{E}_{\epsilon}$ of equilibrium points is continuous at $\epsilon=0$.

Notice that the continuity from the remark above does not exclude the possibility that near an equilibrium point of the limiting equation there may live several different equilibrium points of the perturbed problem. We will actually show that under some additional condition on the differentiability of $h_{\epsilon}$ and assuming that $A_{\epsilon}^{-1} h_{\epsilon}{ }^{\prime}\left(u_{\epsilon}^{*}\right)$ converges compactly to $A^{-1} h_{0}{ }^{\prime}\left(u_{0}^{*}\right)$, where $h_{\epsilon}{ }^{\prime}\left(u_{\epsilon}^{*}\right)$ is the linearization of $h_{\epsilon}$ at $u_{\epsilon}^{*}$, it is possible to obtain the uniqueness of the equilibrium points.

We first prove the $A_{\epsilon}^{-1} h_{\epsilon}{ }^{\prime}\left(u_{\epsilon}^{*}\right)$ converges compactly to $A^{-1} h_{0}{ }^{\prime}\left(u_{0}^{*}\right)$, if $u_{\epsilon}^{*} E$-converges to $u_{0}^{*}$.

Let $h_{\epsilon}{ }^{\prime}\left(u_{\epsilon}^{*}\right): H^{1}\left(\Omega_{\epsilon}\right) \rightarrow H^{-\alpha}\left(\Omega_{\epsilon}\right)$ given by

$$
\left\langle h_{\epsilon}{ }^{\prime}\left(u_{\epsilon}^{*}\right) v_{\epsilon}, w_{\epsilon}\right\rangle=\int_{\Omega_{\epsilon}} \partial_{u} f\left(x, u_{\epsilon}^{*}\right) v_{\epsilon} w_{\epsilon}-\int_{\partial \Omega_{\epsilon}} \partial_{u} g\left(x, u_{\epsilon}^{*}\right) v_{\epsilon} w_{\epsilon}
$$

for $v_{\epsilon} \in H^{1}\left(\Omega_{\epsilon}\right), w_{\epsilon} \in H^{\alpha}\left(\Omega_{\epsilon}\right)$ and $h_{0}{ }^{\prime}\left(u_{0}^{*}\right): H^{1}(\Omega) \rightarrow H^{-\alpha}(\Omega)$ given by

$$
\left\langle h_{0}^{\prime}\left(u_{0}^{*}\right) v, w\right\rangle=\int_{\Omega} \partial_{u} f\left(x, u_{0}^{*}\right) v w-\int_{\partial \Omega} \gamma \partial_{u} g\left(x, u_{0}^{*}\right) v w,
$$

for $v \in H^{1}(\Omega), w \in H^{\alpha}(\Omega)$.

As a matter of fact, we will consider a more general case, defining $\tilde{h}_{\epsilon}$ and $\tilde{h}_{0}$,

$$
\left\langle\tilde{h}_{\epsilon} v_{\epsilon}, w_{\epsilon}\right\rangle=\int_{\Omega_{\epsilon}} V_{\epsilon} v_{\epsilon} w_{\epsilon}+\int_{\partial \Omega_{\epsilon}} b_{\epsilon} v_{\epsilon} w_{\epsilon}, \quad v_{\epsilon} \in H^{1}\left(\Omega_{\epsilon}\right), w_{\epsilon} \in H^{\alpha}\left(\Omega_{\epsilon}\right)
$$

and

$$
\left\langle\tilde{h}_{0} v, w\right\rangle=\int_{\Omega} V_{0} v w+\int_{\partial \Omega} \gamma b_{0} v w, \quad v \in H^{1}(\Omega), w \in H^{\alpha}(\Omega),
$$

where $V_{\epsilon} \in L^{\infty}\left(\Omega_{\epsilon}\right), V_{0} \in L^{\infty}(\Omega), b_{\epsilon} \in L^{\infty}\left(\partial \Omega_{\epsilon}\right), b_{0} \in L^{\infty}(\partial \Omega)$.

Proposition 5.10. Assume conditions $(\mathbf{H})$ and $(\mathbf{F})$ hold. Let $V_{\epsilon}$ and $b_{\epsilon}$ be potentials defined in $\Omega_{\epsilon}$ and in $\partial \Omega_{\epsilon}$ respectively, such that $\left\|V_{\epsilon}\right\|_{L^{\infty}} \leq K$ and $\left\|b_{\epsilon}\right\|_{L^{\infty}} \leq K$. Assume that

$$
\tilde{V}_{\epsilon} \rightarrow \tilde{V}_{0} \text {, weakly in } L^{2}\left(\mathbb{R}^{N}\right) \text {, }
$$

where $\tilde{V}_{\epsilon}$ and $\tilde{V}_{0}$ are the extension by zero of $V_{\epsilon}$ and $V_{0}$ to all of $\mathbb{R}^{N}$. Assume also that for all $i=1,2, \ldots, n$, we have

$$
b_{\epsilon} \circ \phi_{i, \epsilon} \rightarrow b_{0} \circ \phi_{i, 0}, \text { strongly in } L^{2}\left(Q_{N-1}\right)
$$

where $\phi_{i, \epsilon}$ and $\phi_{i, 0}$ are defined in (2.1) and (2.2) respectively. Then, we have $A_{\epsilon}^{-1} \tilde{h}_{\epsilon} \stackrel{C C}{\longrightarrow} A_{0}^{-1} \tilde{h}_{0}$. 
Proof. To show the compact convergence of $A_{\epsilon}^{-1} \tilde{h}_{\epsilon}$ to $A_{0}^{-1} \tilde{h}_{0}$ we follow similar arguments as in the proof of Proposition 5.5 .

i). To show that for fixed $0 \leq \epsilon \leq \epsilon_{0}$, the linear operators $A_{\epsilon}^{-1} \circ \tilde{h}_{\epsilon}$ are compact, we observe that, with elliptic regularity theory, these operators transform $H^{1}\left(\Omega_{\epsilon}\right)$ into $H^{2-\alpha}\left(\Omega_{\epsilon}\right)$, for some $\alpha<1$, which is compactly embedded in $H^{1}\left(\Omega_{\epsilon}\right)$.

ii). Let us consider now a family of functions $u_{\epsilon} \in H^{1}\left(\Omega_{\epsilon}\right)$ with $\left\|u_{\epsilon}\right\|_{H^{1}\left(\Omega_{\epsilon}\right)} \leq C$ and let $w_{\epsilon}=A_{\epsilon}^{-1} \tilde{h}_{\epsilon}\left(u_{\epsilon}\right)$. Notice that this means that $w_{\epsilon}$ is the solution of

$$
\left\{\begin{array}{l}
-\Delta w_{\epsilon}+w_{\epsilon}=V_{\epsilon} u_{\epsilon} \text { in } \Omega_{\epsilon} \\
\frac{\partial w_{\epsilon}}{\partial n}=b_{\epsilon} u_{\epsilon} \quad \text { on } \partial \Omega_{\epsilon}
\end{array}\right.
$$

Multiplying (5.8) by $w_{\epsilon}$, using that $V_{\epsilon}, b_{\epsilon}$ are bounded in $L^{\infty}$ and the uniform boundedness of the trace operator we get, as in Lemma [5.3, that $\left\|w_{\epsilon}\right\|_{H^{1}\left(\Omega_{\epsilon}\right)} \leq C$.

Applying Lemma 4.9 ii), we get subsequences $u_{\epsilon_{k}}, w_{\epsilon_{k}}$ and functions $u, w \in H^{1}(U)$ such that $P_{\Omega_{\epsilon_{k}}, U}\left(u_{\epsilon_{k}}\right) \rightarrow u, P_{\Omega_{\epsilon_{k}}, U}\left(w_{\epsilon_{k}}\right) \rightarrow w$, both w- $H^{1}(U)$ and such that $u_{\epsilon_{k}} \stackrel{E}{\longrightarrow} u_{\mid \Omega}, w_{\epsilon_{k}} \stackrel{E}{\longrightarrow} w_{\mid \Omega}$

Let us show first that $w=A^{-1} \tilde{h}(u)$. For this, observe that if $v \in H^{1}(U)$, then

$$
\left(w_{\epsilon_{k}}, v\right)_{H^{1}\left(\Omega_{\epsilon_{k}}\right)}=\int_{\Omega_{\epsilon_{k}}} V_{\epsilon_{k}} u_{\epsilon_{k}} v+\int_{\partial \Omega_{\epsilon_{k}}} b_{\epsilon_{k}} u_{\epsilon_{k}} v
$$

But from the fact that $\tilde{V}_{\epsilon} \rightarrow \tilde{V}_{0}$ weakly in $L^{2}\left(\mathbb{R}^{N}\right)$ and $\left\|\tilde{V}_{\epsilon}\right\|_{L^{\infty}\left(\mathbb{R}^{N}\right)} \leq K$ then $\tilde{V}_{\epsilon} \rightarrow \tilde{V}_{0}$ weakly in $L^{q}(\Omega)$ for any $1 \leq q<\infty$. From here and using Proposition 4.5 we obtain that

$$
\int_{\Omega_{\epsilon_{k}}} V_{\epsilon_{k}} u_{\epsilon_{k}} v \rightarrow \int_{\Omega} V_{0} u v
$$

For the boundary part,

$$
\begin{aligned}
& \left|\int_{\partial \Omega_{\epsilon_{\epsilon} \cap U_{i}}} b_{\epsilon_{k}} u_{\epsilon_{k}} v-\int_{\partial \Omega \cap U_{i}} \gamma b_{0} u v\right| \\
& \leq\left|\int_{\partial \Omega_{\epsilon_{k}} \cap U_{i}} b_{\epsilon_{k}}\left(u_{\epsilon_{k}}-u\right) v\right|+\left|\int_{\partial \Omega_{\epsilon_{k}} \cap U_{i}} b_{\epsilon_{k}} u v-\int_{\partial \Omega \cap U_{i}} \gamma b_{0} u v\right| \\
& \leq C\left\|u_{\epsilon_{k}}-u\right\|_{L^{2}\left(\partial \Omega_{\epsilon_{k}}\right)}\|v\|_{L^{2}\left(\Omega_{\epsilon_{k}}\right)}+\left|\int_{\partial \Omega_{\epsilon_{k}} \cap U_{i}} b_{\epsilon_{k}} u v-\int_{\partial \Omega \cap U_{i}} \gamma b_{0} u v\right| .
\end{aligned}
$$

But, from Corollary 4.7, we have that the first term in the last expression goes to 0. Moreover, 
for the second term,

$$
\begin{aligned}
& \left|\int_{Q_{N-1}} b_{\epsilon_{k}}\left(\phi_{i, \epsilon_{k}}\right) u\left(\phi_{i, \epsilon_{k}}\right) v\left(\phi_{i, \epsilon_{k}}\right) J \phi_{i, \epsilon_{k}}-\gamma\left(\phi_{i, 0}\right) b_{0}\left(\phi_{i, 0}\right) u\left(\phi_{i, 0}\right) v\left(\phi_{i, 0}\right) J \phi_{i, 0}\right| \\
& =\left|\int_{Q_{N-1}} b_{\epsilon_{k}}\left(\phi_{i, \epsilon_{k}}\right) u\left(\phi_{i, \epsilon_{k}}\right) v\left(\phi_{i, \epsilon_{k}}\right) J \phi_{i, \epsilon_{k}}-b_{0}\left(\phi_{i, 0}\right) u\left(\phi_{i, 0}\right) v\left(\phi_{i, 0}\right) \gamma_{i}\right| \\
& \quad \leq \int_{Q_{N-1}}\left|b_{\epsilon_{k}}\left(\phi_{i, \epsilon_{k}}\right)-b_{0}\left(\phi_{i, 0}\right)\right|\left|u\left(\phi_{i, \epsilon_{k}}\right)\right|\left|v\left(\phi_{i, \epsilon_{k}}\right)\right|\left|J \phi_{i, \epsilon_{k}}\right| \\
& \quad+\int_{Q_{N-1}}\left|b_{0}\left(\phi_{i, 0}\right)\right|\left|u\left(\phi_{i, \epsilon_{k}}\right)-u\left(\phi_{i, 0}\right)\right|\left|v\left(\phi_{i, \epsilon_{k}}\right)\right|\left|J \phi_{i, \epsilon_{k}}\right| \\
& \left.\quad+\int_{Q_{N-1}}\left|b_{0}\left(\phi_{i, 0}\right)\right|\left|u\left(\phi_{i, 0}\right)\right| \mid v\left(\phi_{i, \epsilon_{k}}\right)-v\left(\phi_{i, 0}\right)\right)|| J \phi_{i, \epsilon_{k}} \mid \\
& \quad+\left|\int_{Q_{N-1}} b_{0}\left(\phi_{i, 0}\right) u\left(\phi_{i, 0}\right) v\left(\phi_{i, 0}\right)\left(J \phi_{i, \epsilon_{k}}-\gamma_{i}\right)\right|=I_{1}+I_{2}+I_{3}+I_{4}
\end{aligned}
$$

Since $v, w \in H^{1}(U)$, it follows from Lemma 4.8, that $\int_{Q_{N-1}}\left|v\left(\phi_{i, \epsilon}\right)-v\left(\phi_{i, 0}\right)\right|^{2}$ and $\int_{Q_{N-1}}\left|w\left(\phi_{i, \epsilon}\right)-w\left(\phi_{i, 0}\right)\right|^{2}$ converge to zero when $\epsilon \rightarrow 0$. Using these facts and that $\left\|J \phi_{i, \epsilon}\right\|_{L^{\infty}\left(Q_{N-1}\right)} \leq$ $K$ and $\left\|b_{0}\right\|_{L^{\infty}(\partial \Omega)} \leq K$, we get that $I_{2}, I_{3} \rightarrow 0$.

Also, using Proposition 4.6, we get that $v\left(\phi_{i, \epsilon}\right), w\left(\phi_{i, \epsilon}\right)$ is uniformly bounded in $L^{r}\left(Q_{N-1}\right)$ for $1 \leq r<\frac{N-1}{N-2}$ when $\epsilon \rightarrow 0$. Moreover, $J \phi_{i, \epsilon}$ is uniformly bounded in $L^{\infty}$. Using that $b_{\epsilon} \circ \phi_{i, \epsilon}$ converges to $b_{0} \circ \phi_{i, 0}$ strongly in $L^{p}\left(U_{i} \cap \partial \Omega\right)$ for $1 \leq p<\infty$, we get that $I_{1} \rightarrow 0$.

Finally, using hypothesis (F) and Remark 2.1. $J \phi_{i, \epsilon}$ converges to $\gamma_{i}$ weakly in $L^{p}\left(Q_{N-1}\right)$, for any $1 \leq p<\infty$. This implies that $I_{4} \rightarrow 0$.

Hence, passing to the limit in both sides of (5.9) we have that

$$
(w, v)_{H^{1}(\Omega)}=\int_{\Omega} V_{0} u v+\int_{\partial \Omega} \gamma b_{0} u v
$$

which shows that $w=A^{-1} \tilde{h}_{0}(u)$.

Let us show now that, as a matter of fact, $w_{\epsilon_{k}} \stackrel{E}{\longrightarrow} w$. From Proposition 3.4 it will be enough to show that $\left\|w_{\epsilon_{k}}\right\|_{H^{1}\left(\Omega_{\epsilon_{k}}\right)} \rightarrow\|w\|_{H^{1}(\Omega)}$. But,

$$
\left\|w_{\epsilon_{k}}\right\|_{H^{1}\left(\Omega_{\epsilon_{k}}\right)}^{2}=\int_{\Omega_{\epsilon_{k}}} V_{\epsilon_{k}} u_{\epsilon_{k}} w_{\epsilon_{k}}+\int_{\partial \Omega_{\epsilon_{k}}} b_{\epsilon_{k}} u_{\epsilon_{k}} w_{\epsilon_{k}}
$$

and with a very similar argument as the one above, we can show that

$$
\int_{\Omega_{\epsilon_{k}}} V_{\epsilon_{k}} u_{\epsilon_{k}} w_{\epsilon_{k}}+\int_{\partial \Omega_{\epsilon_{k}}} b_{\epsilon_{k}} u_{\epsilon_{k}} w_{\epsilon_{k}} \rightarrow \int_{\Omega} V_{0} u w+\int_{\partial \Omega} \gamma b_{0} u w=\|w\|_{H^{1}(\Omega)}
$$

which shows the convergence of the $H^{1}$ norms.

iii) To prove that if $u_{\epsilon} \stackrel{E}{\longrightarrow} u$ then $w_{\epsilon} \stackrel{E}{\longrightarrow} w$ we follow the same line of proof as it was done to show iii) in the proof of Proposition 5.5.

Hence, we can show easily now the following 
Corollary 5.11. Let $u_{\epsilon}^{*}$ and $u_{0}^{*}$ be equilibrium points of (1.1) and (1.2) respectively such that $u_{\epsilon}^{*} \stackrel{E}{\longrightarrow} u_{0}^{*}$. Let $h_{\epsilon}{ }^{\prime}\left(u_{\epsilon}^{*}\right)$ and $h_{0}{ }^{\prime}\left(u_{0}^{*}\right)$ be given by (5.4) and (5.5). Then, the sequence of operators $A_{\epsilon}^{-1} h_{\epsilon}{ }^{\prime}\left(u_{\epsilon}^{*}\right) \in \mathcal{L}\left(H^{1}\left(\Omega_{\epsilon}\right)\right)$ converges compactly to $A^{-1} h_{0}{ }^{\prime}\left(u_{0}^{*}\right) \in \mathcal{L}\left(H^{1}(\Omega)\right)$.

In particular, we can apply Proposition 3.10.

Proof. We just apply Proposition 5.10 with $V_{\epsilon}(x)=\frac{\partial f}{\partial u}\left(x, u_{\epsilon}^{*}(x)\right), b_{\epsilon}(x)=\frac{\partial g}{\partial u}\left(x, u_{\epsilon}^{*}(x)\right)$ for $0 \leq \epsilon \leq \epsilon_{0}$, which satisfy the hypotheses of Proposition [5.10.

We also want to show the uniqueness of the solutions given in Theorem 2.4. For this, we will need the following

Lemma 5.12. Assume $f, g$ satisfy 2.4) and (2.5). There exists $K>0$ such that for all $v_{\epsilon}$, $\left\|v_{\epsilon}\right\|_{H^{1}\left(\Omega_{\epsilon}\right)} \leq 1$ we have

$$
\left\|A_{\epsilon}^{-1}\left(h_{\epsilon}\left(u_{\epsilon}^{*}+v_{\epsilon}\right)-h_{\epsilon}\left(u_{\epsilon}^{*}\right)-h_{\epsilon}^{\prime}\left(u_{\epsilon}^{*}\right) v_{\epsilon}\right)\right\|_{H^{1}\left(\Omega_{\epsilon}\right)} \leq K\left\|v_{\epsilon}\right\|_{H^{1}\left(\Omega_{\epsilon}\right)}^{1+\frac{1}{N-1}}
$$

Proof. Let $w_{\epsilon}=A_{\epsilon}^{-1}\left(h_{\epsilon}\left(u_{\epsilon}^{*}+v_{\epsilon}\right)-h_{\epsilon}\left(u_{\epsilon}^{*}\right)-h_{\epsilon}^{\prime}\left(u_{\epsilon}^{*}\right) v_{\epsilon}\right)$. This means $A_{\epsilon} w_{\epsilon}=h_{\epsilon}\left(u_{\epsilon}^{*}+v_{\epsilon}\right)-$ $h_{\epsilon}\left(u_{\epsilon}^{*}\right)-h_{\epsilon}^{\prime}\left(u_{\epsilon}^{*}\right) v_{\epsilon}$, and $w_{\epsilon}$ is a solution of

$$
\left\{\begin{array}{l}
-\Delta w_{\epsilon}+w_{\epsilon}=f\left(x, u_{\epsilon}^{*}+v_{\epsilon}\right)-f\left(x, u_{\epsilon}^{*}\right)-\partial_{u} f\left(x, u_{\epsilon}^{*}\right) v_{\epsilon}, \text { in } \Omega_{\epsilon}, \\
\frac{\partial w_{\epsilon}}{\partial n}+g\left(x, u_{\epsilon}^{*}+v_{\epsilon}\right)-g\left(x, u_{\epsilon}^{*}\right)-\partial_{u} g\left(x, u_{\epsilon}\right) v_{\epsilon}=0 \text { on } \partial \Omega_{\epsilon} .
\end{array}\right.
$$

Multiplying the equation by $w_{\epsilon}$ and integrating by parts, we get

$$
\begin{aligned}
& \left.\left\|w_{\epsilon}\right\|_{H^{1}\left(\Omega_{\epsilon}\right)}^{2}=\int_{\Omega_{\epsilon}}\left(f\left(x, u_{\epsilon}^{*}+v_{\epsilon}\right)-f\left(x, u_{\epsilon}^{*}\right)-\partial_{u} f\left(x, u_{\epsilon}^{*}\right) v_{\epsilon}\right)\right) w_{\epsilon} \\
& \left.-\int_{\partial \Omega_{\epsilon}}\left(g\left(x, u_{\epsilon}^{*}+v_{\epsilon}\right)-g\left(x, u_{\epsilon}^{*}\right)-\partial g\left(x, u_{\epsilon}^{*}\right) v_{\epsilon}\right)\right) w_{\epsilon} .
\end{aligned}
$$

We will show that

$$
\left.\mid \int_{\Omega_{\epsilon}}\left(f\left(x, u_{\epsilon}^{*}+v_{\epsilon}\right)-f\left(x, u_{\epsilon}^{*}\right)-\partial f\left(x, u_{\epsilon}^{*}\right) v_{\epsilon}\right)\right) w_{\epsilon} \mid \leq \tilde{C}_{\eta}\left\|v_{\epsilon}\right\|_{H^{1}}^{2+4 / N}+\eta\left\|w_{\epsilon}\right\|_{H^{1}\left(\Omega_{\epsilon}\right)}^{2}
$$

and

$$
\left.\mid \int_{\partial \Omega_{\epsilon}}\left(g\left(x, u_{\epsilon}^{*}+v_{\epsilon}\right)-g\left(x, u_{\epsilon}^{*}\right)-\partial g\left(x, u_{\epsilon}^{*}\right) v_{\epsilon}\right)\right) w_{\epsilon} \mid \leq \tilde{C}_{\eta}\left\|v_{\epsilon}\right\|_{H^{1}}^{2+2 /(N-1)}+\eta\left\|w_{\epsilon}\right\|_{H^{1}\left(\Omega_{\epsilon}\right)}^{2},
$$

for some $\eta<1$.

In fact, using Hölder and Young inequalities we have

$$
\begin{aligned}
\mid \int_{\Omega_{\epsilon}}\left(f\left(x, u_{\epsilon}^{*}+v_{\epsilon}\right)-f\left(x, u_{\epsilon}^{*}\right)\right. & \left.\left.-\partial_{u} f\left(x, u_{\epsilon}^{*}\right) v_{\epsilon}\right)\right) w_{\epsilon} \mid \\
& \left.\leq \| f\left(\cdot, u_{\epsilon}^{*}+v_{\epsilon}\right)-f\left(\cdot, u_{\epsilon}^{*}\right)-\partial_{u} f\left(\cdot, u_{\epsilon}^{*}\right) v_{\epsilon}\right)\left\|_{L^{2}\left(\Omega_{\epsilon}\right)}\right\| w_{\epsilon} \|_{L^{2}\left(\Omega_{\epsilon}\right)} \\
& \left.\leq C_{\eta} \| f\left(\cdot, u_{\epsilon}^{*}+v_{\epsilon}\right)-f\left(\cdot, u_{\epsilon}^{*}\right)-\partial_{u} f\left(\cdot, u_{\epsilon}^{*}\right) v_{\epsilon}\right)\left\|_{L^{2}\left(\Omega_{\epsilon}\right)}^{2}+\eta\right\| w_{\epsilon} \|_{L^{2}\left(\Omega_{\epsilon}\right)}^{2} .
\end{aligned}
$$


Since the function $f$ satisfies (2.4), we can show that, if we define $\theta_{\epsilon}(x)=\min \left\{1,\left|v_{\epsilon}(x)\right|\right\}$, then

$$
\left.\mid f\left(x, u_{\epsilon}^{*}(x)+v_{\epsilon}(x)\right)-f\left(x, u_{\epsilon}^{*}(x)\right)-\partial_{u} f\left(x, u_{\epsilon}^{*}(x)\right) v_{\epsilon}(x)\right)\left|\leq C_{1} \theta_{\epsilon}(x)\right| v_{\epsilon}(x) \mid, \text { ae } x \in \Omega_{\epsilon},
$$

for some constant $C_{1}$ independent of $\epsilon, v_{\epsilon}$ and $u_{\epsilon}^{*}$. To see this, notice that applying the mean value theorem, we have $\left.\mid f\left(x, u_{\epsilon}^{*}(x)+v_{\epsilon}(x)\right)-f\left(x, u_{\epsilon}^{*}(x)\right)-\partial_{u} f\left(x, u_{\epsilon}^{*}(x)\right) v_{\epsilon}(x)\right) \mid=$ $\left|\left(\partial_{u} f\left(x, \tilde{u}_{\epsilon}(x)\right)-\partial_{u} f\left(x, u_{\epsilon}(x)\right)\right) v_{\epsilon}(x)\right|$, where $\tilde{u}_{\epsilon}(x)$ is certain intermediate point between $u_{\epsilon}^{*}(x)$ and $u_{\epsilon}^{*}(x)+v_{\epsilon}(x)$. Hence, by the uniform boundedness of $\partial_{u} f(x, u)$ we have that $\left|\partial_{u} f\left(x, \tilde{u}_{\epsilon}(x)\right)-\partial_{u} f\left(x, u_{\epsilon}(x)\right)\right| \leq 2 C$. On the other hand, using again the mean value theorem and the uniform boundedness of $\partial_{u u} f$, we get $\left|\partial_{u} f\left(x, \tilde{u}_{\epsilon}(x)\right)-\partial_{u} f\left(x, u_{\epsilon}^{*}(x)\right)\right|=$ $\left|\partial_{u u} f\left(x, \bar{u}_{\epsilon}(x)\right)\right| \tilde{u}_{\epsilon}(x)-u_{\epsilon}^{*}(x)|\leq C| v(x) \mid$, for some value $\bar{u}_{\epsilon}(x)$ between $\tilde{u}_{\epsilon}(x)$ and $u_{\epsilon}^{*}(x)$. This implies that $\left|\partial_{u} f\left(x, \tilde{u}_{\epsilon}(x)\right)-\partial_{u} f\left(x, u_{\epsilon}(x)\right)\right| \leq 2 C \min \left\{1, v_{\epsilon}(x)\right\}$, from where the result follows.

This implies that $\left\|\theta_{\epsilon}\right\|_{L^{\infty}\left(\Omega_{\epsilon}\right)} \leq 1$ and $\left\|\theta_{\epsilon}\right\|_{L^{2}\left(\Omega_{\epsilon}\right)} \leq\left\|v_{\epsilon}\right\|_{L^{2}\left(\Omega_{\epsilon}\right)}$. By interpolation, we get $\left\|\theta_{\epsilon}\right\|_{L^{p}\left(\Omega_{\epsilon}\right)} \leq\left\|v_{\epsilon}\right\|_{L^{2}\left(\Omega_{\epsilon}\right)}^{2 / p}$, for any $2 \leq p<\infty$.

Thus, using that the embedding $H^{1}\left(\Omega_{\epsilon}\right) \hookrightarrow L^{q}\left(\Omega_{\epsilon}\right)$ is uniformly bounded for $1 \leq q \leq$ $2 N /(N-2)$, we get,

$$
\begin{gathered}
\left.\| f\left(u_{\epsilon}^{*}+v_{\epsilon}\right)-f\left(u_{\epsilon}^{*}\right)-f^{\prime}\left(u_{\epsilon}^{*}\right) v_{\epsilon}\right)\left\|_{L^{2}\left(\Omega_{\epsilon}\right)}^{2} \leq C_{1}\right\| \theta_{\epsilon} v_{\epsilon} \|_{L^{2}\left(\Omega_{\epsilon}\right)}^{2} \\
\leq C_{1}\left\|\theta_{\epsilon}\right\|_{L^{N}\left(\Omega_{\epsilon}\right)}^{2}\left\|v_{\epsilon}\right\|_{L^{\frac{2 N}{N-2}}\left(\Omega_{\epsilon}\right)}^{2} \leq C_{1}\left\|v_{\epsilon}\right\|_{H^{1}\left(\Omega_{\epsilon}\right)}^{2+4 / N}
\end{gathered}
$$

which shows (5.12).

For the boundary part, using Hölder and Young inequalities we have

$$
\begin{aligned}
\mid \int_{\partial \Omega_{\epsilon}}\left(g\left(u_{\epsilon}^{*}+v_{\epsilon}\right) g\left(u_{\epsilon}^{*}\right)\right. & \left.\left.\left.-g^{\prime}\left(u_{\epsilon}^{*}\right) v_{\epsilon}\right)\right) w_{\epsilon} \mid \leq \| g\left(u_{\epsilon}^{*}+v_{\epsilon}\right)-g\left(u_{\epsilon}^{*}\right)-g^{\prime}\left(u_{\epsilon}^{*}\right) v_{\epsilon}\right)\left\|_{L^{2}\left(\partial \Omega_{\epsilon}\right)}\right\| w_{\epsilon} \|_{L^{2}\left(\partial \Omega_{\epsilon}\right)} \\
& \left.\leq C_{\eta} \| g\left(u_{\epsilon}^{*}+v_{\epsilon}\right)-g\left(u_{\epsilon}^{*}\right)-g^{\prime}\left(u_{\epsilon}^{*}\right) v_{\epsilon}\right)\left\|_{L^{2}\left(\partial \Omega_{\epsilon}\right)}^{2}+\eta\right\| w_{\epsilon} \|_{L^{2}\left(\partial \Omega_{\epsilon}\right)}^{2} .
\end{aligned}
$$

Using the uniform imbedding of $H^{1}\left(\Omega_{\epsilon}\right)$ in $L^{2}\left(\partial \Omega_{\epsilon}\right)$, we get

$\left.\left.\mid \int_{\partial \Omega_{\epsilon}}\left(g\left(u_{\epsilon}^{*}+v_{\epsilon}\right) g\left(u_{\epsilon}^{*}\right)-g^{\prime}\left(u_{\epsilon}^{*}\right) v_{\epsilon}\right)\right) w_{\epsilon} \mid \leq C_{\eta} \| g\left(u_{\epsilon}^{*}+v_{\epsilon}\right)-g\left(u_{\epsilon}^{*}\right)-g^{\prime}\left(u_{\epsilon}^{*}\right) v_{\epsilon}\right)\left\|_{L^{2}\left(\partial \Omega_{\epsilon}\right)}^{2}+\eta\right\| w_{\epsilon} \|_{H^{1}\left(\Omega_{\epsilon}\right)}^{2}$

As we did above for $f$, we can also prove that,

$$
\left.\mid g\left(u_{\epsilon}^{*}(x)+v_{\epsilon}(x)\right)-g\left(u_{\epsilon}^{*}(x)\right)-g^{\prime}\left(u_{\epsilon}^{*}(x)\right) v_{\epsilon}(x)\right)\left|\leq C_{2} \theta_{\epsilon}(x)\right| v_{\epsilon}(x) \mid,
$$

where $\theta_{\epsilon}(x)=\min \left\{1,\left|v_{\epsilon}(x)\right|\right\}$ for $x$ ae in $\partial \Omega_{\epsilon}$, then $\left\|\theta_{\epsilon}\right\|_{L^{\infty}} \leq 1$ and $\left\|\theta_{\epsilon}\right\|_{L^{2}\left(\partial \Omega_{\epsilon}\right)} \leq\left\|v_{\epsilon}\right\|_{L^{2}\left(\partial \Omega_{\epsilon}\right)}$. By interpolation, we get $\left\|\theta_{\epsilon}\right\|_{L^{p}\left(\partial \Omega_{\epsilon}\right)} \leq\left\|v_{\epsilon}\right\|_{L^{2}\left(\partial \Omega_{\epsilon}\right)}^{2 / p}$, for $2 \leq p<\infty$.

Thus, with the analysis above and using that the trace operator $H^{1}\left(\Omega_{\epsilon}\right) \rightarrow L^{q}\left(\partial \Omega_{\epsilon}\right)$ is uniformly bounded in $\epsilon$ for $1 \leq q \leq \frac{2(N-1)}{N-2}$ we get

$$
\begin{aligned}
\| g\left(u_{\epsilon}^{*}+v_{\epsilon}\right)-g\left(u_{\epsilon}^{*}\right) & \left.-g^{\prime}\left(u_{\epsilon}^{*}\right) v_{\epsilon}\right)\left\|_{L^{2}\left(\partial \Omega_{\epsilon}\right)}^{2} \leq C_{2}\right\| \theta_{\epsilon} v_{\epsilon} \|_{L^{2}\left(\partial \Omega_{\epsilon}\right)}^{2} \\
& \leq C_{2}\left\|\theta_{\epsilon}\right\|_{L^{2(N-1)}\left(\partial \Omega_{\epsilon}\right)}^{2}\left\|v_{\epsilon}\right\|_{L^{\frac{2(N-1)}{N-2}\left(\partial \Omega_{\epsilon}\right)}}^{2} \leq C_{2}\left\|v_{\epsilon}\right\|_{H^{1}\left(\Omega_{\epsilon}\right)}^{2+2 /(N-1)}
\end{aligned}
$$


which shows (5.13).

Plugging (5.12) and (5.13) in (5.11), choosing $\eta<1 / 2$ and realizing that for $N \geq 2$ we have $1 /(N-1) \leq 2 / N$, we show the Lemma.

Now, we prove the uniqueness result of the main theorem.

Proposition 5.13. If $u_{0}^{*}$ is a hyperbolic equilibrium point of (1.2), then there exist $\delta>0$ and $\epsilon_{0}>0$ such that for $0<\epsilon<\epsilon_{0}$ there exists one and only one equilibrium point $u_{\epsilon}^{*}$ of (1.1), with $\left\|u_{\epsilon}^{*}-E_{\epsilon} u_{0}^{*}\right\| \leq \delta$.

Proof. The existence of at least one equilibrium $u_{\epsilon}^{*}$ with $\left\|u_{\epsilon}^{*}-E_{\epsilon} u_{0}^{*}\right\| \leq \delta$ is guaranteed by Corollary 5.8. Now we prove that $u_{\epsilon}^{*}$ is unique. In fact, $u_{\epsilon}$ is a equilibrium point of (1.1) if and only if $u_{\epsilon}$ is a fixed point of $A_{\epsilon}^{-1} h_{\epsilon}$. We prove that if $u_{\epsilon} \neq u_{\epsilon}^{*}$ and $\left\|u_{\epsilon}-u_{\epsilon}^{*}\right\| \leq \delta$ for some $\delta>0$, then

$$
\left\|u_{\epsilon}-A_{\epsilon}^{-1} h_{\epsilon}\left(u_{\epsilon}\right)\right\|>0 .
$$

Since $u_{0}^{*}$ is a hyperbolic equilibrium, we get that $\left\|\left(I-A_{0}^{-1} h_{0}^{\prime}\left(u_{0}^{*}\right)\right)^{-1}\right\| \leq M$. Moreover, $A_{\epsilon}^{-1} h_{\epsilon}^{\prime}\left(u_{\epsilon}^{*}\right)$ converges compactly to $A^{-1} h_{0}^{\prime}\left(u_{0}^{*}\right)$. This implies by Lemma 3.9 that $\|(I-$ $\left.A_{\epsilon}^{-1} h_{\epsilon}^{\prime}\left(u_{\epsilon}^{*}\right)\right)^{-1} \| \leq M, M$ independent of $\epsilon$. In particular, there exists $\eta>0$ such that $\left\|\left(I-A_{\epsilon}^{-1} h_{\epsilon}^{\prime}\left(u_{\epsilon}^{*}\right)\right)\left(z_{\epsilon}\right)\right\| \geq \eta\left\|z_{\epsilon}\right\|$. Thus,

$$
\begin{aligned}
\left\|u_{\epsilon}-A_{\epsilon}^{-1} h_{\epsilon}\left(u_{\epsilon}\right)\right\|_{H^{1}\left(\Omega_{\epsilon}\right)} & \geq\left\|u_{\epsilon}-u_{\epsilon}^{*}-A_{\epsilon} h_{\epsilon}^{\prime}\left(u_{\epsilon}^{*}\right)\left(u_{\epsilon}-u_{\epsilon}^{*}\right)\right\|_{H^{1}\left(\Omega_{\epsilon}\right)} \\
& -\left\|A_{\epsilon}^{-1}\left(h\left(u_{\epsilon}\right)-h\left(u_{\epsilon}^{*}\right)-h^{\prime}\left(u_{\epsilon}^{*}\right)\left(u_{\epsilon}-u_{\epsilon}^{*}\right)\right)\right\|_{H^{1}\left(\Omega_{\epsilon}\right)} .
\end{aligned}
$$

By Lemma [5.12] we have that there exists $0<\delta_{0}<1$ such that

$$
\left\|A_{\epsilon}^{-1}\left(h\left(u_{\epsilon}\right)-h\left(u_{\epsilon}^{*}\right)-h^{\prime}\left(u_{\epsilon}^{*}\right)\left(u_{\epsilon}-u_{\epsilon}^{*}\right)\right)\right\|_{H^{1}\left(\Omega_{\epsilon}\right)} \leq \eta / 2\left\|\left(u_{\epsilon}-u_{\epsilon}^{*}\right)\right\|_{H^{1}\left(\Omega_{\epsilon}\right)},
$$

for $\left\|\left(u_{\epsilon}-u_{\epsilon}^{*}\right)\right\|_{H^{1}\left(\Omega_{\epsilon}\right)} \leq \delta_{0}$. Using this, we get that

$$
\left\|u_{\epsilon}-A_{\epsilon}^{-1} h_{\epsilon}\left(u_{\epsilon}\right)\right\|_{H^{1}\left(\Omega_{\epsilon}\right)} \geq \eta / 2\left\|u_{\epsilon}-u_{\epsilon}^{*}\right\|_{H^{1}\left(\Omega_{\epsilon}\right)} .
$$

and this completes the proof.

With all the results obtained in this section, we can easily provide a proof of the two main results.

Proof of Theorem [2.4; Part i) is obtained from Corollary 5.6] and Proposition [5.7. Part ii) is proved from Proposition 5.13 and from the convergence in $C^{\beta}$ (and therefore in $L^{\infty}$ ) obtained in Part i).

Proof of Theorem 2.6: We just need to apply Corollary 5.11]

\section{References}

[1] H. Amann, Linear and Quasilinear Parabolic Problems. Abstract Linear Theory. Birkäuser Verlag, (1995). 
[2] J. M. Arrieta and S. M. Bruschi, Boundary oscillations and nonlinear boundary conditions, C.R. Acad. Sc. Paris, series I, t. 343, pp. 99-104 (2006)

[3] J. M. Arrieta and S. M. Bruschi, Rapidly varying boundaries in equations with nonlinear boundary conditions. The case of a non-lipschitz deformation. In preparation.

[4] J. M. Arrieta and A. N. Carvalho, Spectral convergence and nonlinear dynamics of reaction-diffusion equations under perturbations of domain, Journal of Differential Equations 199, (2004) 143-178.

[5] J. M. Arrieta, A. N. Carvalho and G. Lozada-Cruz, Dynamics in dumbbell domains I. Continuity of the set of equilibria., J. Differential Equations, To appear.

[6] J. M. Arrieta, A. N. de Carvalho and A. Rodriguez-Bernal, Parabolic problems with nonlinear boundary conditions and critical nonlinearities, Journal of Differential Equations 156, (1999) 376-406.

[7] J. M. Arrieta, A. N. de Carvalho and A. Rodriguez-Bernal, Attractors of parabolic problems with nonlinear boundary conditions: Uniform bounds. Comm. Partial Differential Equations 25 (2000), 1-37.

[8] H. Brézis, Análisis funcional, Teoría y aplicaciones, Alianza Universidad Textos, Madrid, 1984.

[9] A. Carvalho, S. Piskarev, A general approximation scheme for attractors of abstract parabolic problems, Numer. Funct. Anal. Optim. To appear.

[10] J. Casado-Díaz, E. Fernández-Cara, J. Simon, Why viscous fluids adhere to rugose walls: a mathematical explanation, Journal of Differential Equations 189 (2003), no. $2,526-537$.

[11] G. Chechkin, A. Friedman, A.L. Piatnitski, The boundary-value problem in domains with very rapidly oscillating boundary, J.of Math. Anal. Appl. 231, pp. 213-234 (1999)

[12] E. N. Dancer and D. Daners, Domain Perturbations for Elliptic Equations subject to Robin Boundary Conditions, Journal of Differential Equations 138, (1997) 86-132.

[13] M.A. Krasnoselskii, P.P. Zabreiko, Geometrical Methods of Nonlinear Analysis (Translated from the Russian by C.C. Fenske) Grundlehren Math. Wiss (Fundamental Principles of Mathematical Sciences) vol 263, Springer, Berlin 1984.

[14] O. Ladyzhenskaya and N. N. Ural'tseva, Linear and Quasilinear Ellpitic Equations, Academic Press, (1968).

[15] V.G. Maz’ja, Sobolev Spaces, Springer-Verlag, Berlin 1985 
[16] N. Neuss, M. Neuss-Radu, A. Mikelic, Effective laws for the Poisson equation on domains with curved osicllating boundaries, Preprint 2004-36, SFB 359, Heidelberg.

[17] S.E. Pastukhova, The oscillating boundary phenomenon in the homogenization of a climatization problem, Differential Equations 37, pp 1276-1283 (2001)

[18] E. Sanchez-Palencia, Non homogeneous media and vibration theory, Volume 127, Lecture Notes in Physics, Springer-Verlag, Berlin (1980)

[19] G. Vainikko, Approximative methods for nonlinear equations (two approaches to the convergence problem), Nonlinear Analysis, Theory, Methods \& Applications, vol2, 6 (1978) 647-687. 